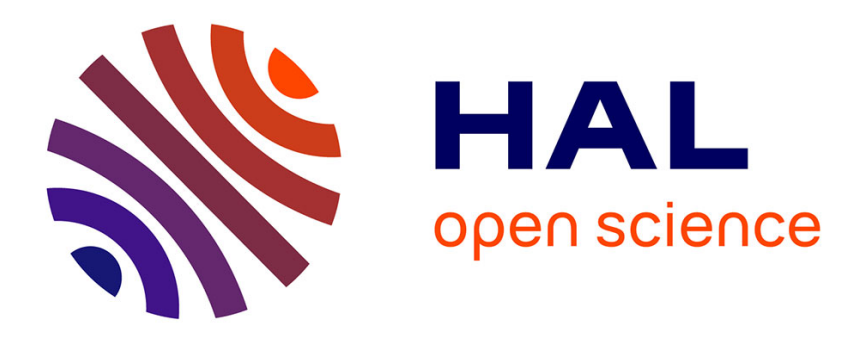

\title{
Ergodic problems for viscous Hamilton-Jacobi equations with inward drift
}

\author{
Emmanuel Chasseigne, Naoyuki Ichihara
}

\section{To cite this version:}

Emmanuel Chasseigne, Naoyuki Ichihara. Ergodic problems for viscous Hamilton-Jacobi equations with inward drift. SIAM Journal on Control and Optimization, 2019, 57 (1), pp.23-52. 10.1137/18M1179328. hal-01864453

\section{HAL Id: hal-01864453 \\ https://hal.science/hal-01864453}

Submitted on 29 Aug 2018

HAL is a multi-disciplinary open access archive for the deposit and dissemination of scientific research documents, whether they are published or not. The documents may come from teaching and research institutions in France or abroad, or from public or private research centers.
L'archive ouverte pluridisciplinaire HAL, est destinée au dépôt et à la diffusion de documents scientifiques de niveau recherche, publiés ou non, émanant des établissements d'enseignement et de recherche français ou étrangers, des laboratoires publics ou privés. 


\title{
ERGODIC PROBLEMS FOR VISCOUS HAMILTON-JACOBI EQUATIONS WITH INWARD DRIFT
}

\author{
EMMANUEL CHASSEIGNE* $^{*}$ AND NAOYUKI ICHIHARA ${ }^{\dagger}$
}

\begin{abstract}
In this paper we study the ergodic problem for viscous Hamilton-Jacobi equations with superlinear Hamiltonian and inward drift. We investigate (i) existence and uniqueness of eigenfunctions associated with the generalized principal eigenvalue of the ergodic problem, (ii) relationships with the corresponding stochastic control problem of both finite and infinite time horizon, and (iii) the precise growth exponent of the generalized principal eigenvalue with respect to a perturbation of the potential function.
\end{abstract}

Key words. Ergodic problem, viscous Hamilton-Jacobi equation, stochastic ergodic control, generalized principal eigenvalue.

AMS subject classifications. 35Q93; 60J60; 93E20

1. Introduction. This paper is concerned with the ergodic problem for viscous HamiltonJacobi equations of the form

$$
\lambda-\frac{1}{2} \Delta \phi(x)+b(x) \cdot D \phi(x)+H(x, D \phi(x))-V(x)=0, \quad x \in \mathbf{R}^{d},
$$

where $b: \mathbf{R}^{d} \rightarrow \mathbf{R}^{d}, H: \mathbf{R}^{d} \times \mathbf{R}^{d} \rightarrow \mathbf{R}$, and $V: \mathbf{R}^{d} \rightarrow \mathbf{R}$ are given functions, $D \phi$ and $\Delta \phi$ are, respectively, the gradient and the Laplacian of $\phi: \mathbf{R}^{d} \rightarrow \mathbf{R}$, and " . "stands for the inner product in $\mathbf{R}^{d}$. Throughout the paper, any pair $(\lambda, \phi) \in \mathbf{R} \times C^{2}\left(\mathbf{R}^{d}\right)$ satisfying (EP) is called a solution of $(\mathrm{EP})$, and $\lambda, \phi$ are referred to as an eigenvalue of (EP) and an eigenfunction of (EP) associated with $\lambda$, respectively.

The main results of this paper consist of three parts. In order to state them briefly, we assume in this introductory section that

$$
b(x)=\left(1+|x|^{2}\right)^{\frac{\delta-1}{2}} x, \quad H(x, p)=\frac{1}{m}|p|^{m}, \quad V(x)=\left(1+|x|^{2}\right)^{-\frac{\eta}{2}},
$$

where $\delta \in(0,1], m>1$, and $\eta>0$ are given constants. More precise (and more general) assumptions on $(b, H, V)$ will be stated in the next section. The crucial properties in (1.1) are:

(i) drift vector $-b(x)$ is inward-pointing with the intensity of order $|x|^{\delta}$,

(ii) Hamiltonian $H(x, p)$ is convex in $p$ and diverges as $|p| \rightarrow \infty$ with the order $|p|^{m}$,

(iii) potential $V(x)$ is positive and vanishes as $|x| \rightarrow \infty$ with the order $|x|^{-\eta}$.

In the first part of this paper, we discuss the existence and uniqueness of eigenfunctions $\phi$ of (EP) associated with the generalized principal eigenvalue $\lambda_{\max }$ defined by

$$
\lambda_{\max }:=\sup \left\{\lambda \in \mathbf{R} \mid(\lambda, \phi) \in \mathbf{R} \times C^{3}\left(\mathbf{R}^{d}\right) \text { is a subsolution of }(\mathrm{EP})\right\}
$$

where $(\lambda, \phi)$ is said to be a subsolution (resp. supersolution) of (EP) if the left-hand side of (EP) is nonpositive (resp. nonnegative) for all $x \in \mathbf{R}^{d}$. In Theorem 2.1 we prove that there exists a unique eigenfunction $\phi^{*} \in C^{2}\left(\mathbf{R}^{d}\right)$ of $(\mathrm{EP})$ associated with $\lambda_{\max }$, up to an additive constant. The solvability of (EP), especially, the uniqueness of eigenfunctions associated with the generalized principal eigenvalue has been studied under a variety of conditions on $(b, H, V)$ (see, e.g. $[3,4,10$, $17,18,19,20,21,22]$ and the references therein). Our result can be regarded as extensions of $[4,18]$ and $[17,21,22]$, the former of which deal with ergodic problems without drift (i.e., $b(x) \equiv 0)$, while the latter restrict their attention to quadratic Hamiltonians (i.e., $m=2$ ). We mention that [10]

\footnotetext{
*Institut Denis Poisson (UMR CNRS 7013), Université de Tours, Parc de Grandmont, 37200 Tours, France. Email: emmanuel.chasseigne@lmpt.univ-tours.fr.

${ }^{\dagger}$ Department of Physics and Mathematics, Aoyama Gakuin University, 5-10-1 Fuchinobe, Chuo-ku, Sagamiharashi, Kanagawa 252-5258, Japan. Email: ichihara@gem.aoyama.ac.jp.
} 
studies existence of eigenfunctions of (EP) under mild assumptions including (1.1) by a different argument. However, uniqueness is not discussed there.

In the second part, we investigate some relationships between the solution $\left(\lambda_{\max }, \phi^{*}\right)$ of $(\mathrm{EP})$ obtained in the first part and the corresponding stochastic optimal control problem of both finite and infinite time horizon. More specifically, let $\xi=\left(\xi_{t}\right)_{t \geq 0}$ be an $\mathbf{R}^{d}$-valued control process belonging to an admissible class $\mathcal{A}$ specified later. Let $X^{\xi}=\left(X_{t}^{\xi}\right)_{t \geq 0}$ be the controlled process governed by the stochastic differential equation

$$
d X_{t}^{\xi}=-\xi_{t} d t-b\left(X_{t}^{\xi}\right) d t+d W_{t}, \quad t \geq 0,
$$

where $W=\left(W_{t}\right)_{t \geq 0}$ stands for a $d$-dimensional standard Brownian motion defined on some probability space. We set

$$
J(T, x ; \xi):=\mathbf{E}_{x}\left[\int_{0}^{T}\left(\frac{1}{m^{*}}\left|\xi_{t}\right|^{m^{*}}+V\left(X_{t}^{\xi}\right)\right) d t\right], \quad T>0, \quad x \in \mathbf{R}^{d},
$$

where $m^{*}:=m /(m-1)$ and $\mathbf{E}_{x}$ denotes the expectation conditioned on $X_{0}^{\xi}=x$. Then we prove in Theorem 2.2 that the value function of the long-run average $\Lambda(x):=\inf _{\xi \in \mathcal{A}} \limsup _{T \rightarrow \infty}(1 / T) J(T, x ; \xi)$ coincides with $\lambda_{\max }$ for all $x \in \mathbf{R}^{d}$, and that the value function of finite time horizon $T$ defined by $u(T, x):=\inf _{\xi \in \mathcal{A}} J(T, x ; \xi)$ satisfies

$$
u(T, \cdot)-\lambda_{\max } T \longrightarrow \phi^{*}+c \quad \text { as } T \rightarrow \infty
$$

for some $c \in \mathbf{R}$ on any compact subset of $\mathbf{R}^{d}$. Although convergence (1.5) is natural to expect, its validity is not trivial at all since the value of the constant $c$ in (1.5) cannot be determined by ergodic problem (EP), only. The asymptotic behavior of the value function $u(T, x)$ as time horizon $T$ tends to infinity has been investigated, from both PDE and stochastic control point of view, in various settings (see, e.g. [2, 5, 6, 7, 8, 11, 12, 14, 16, 18, 21, 25, 28, 31, 32] and references therein). The present paper can be compared with $[18,21]$, where either $b=0$ or $m=2$ is imposed. Note that equality $\Lambda(x)=\lambda_{\max }$ has been obtained in [10], but convergence (1.5) seems to be new.

The third part of this paper concerns certain asymptotic properties of the generalized principal eigenvalue $\lambda_{\max }$ with respect to a perturbation of $V$; we introduce a real parameter $\beta \geq 0$ and consider (EP) with $\beta V$ in place of $V$. Then we easily see that the function $\beta \rightarrow \lambda_{\max }(\beta)$ is nondecreasing and concave. Such kind of qualitative properties of $\lambda_{\max }(\beta)$ with respect to $\beta$ have been studied by $[9,19,20]$ in connection with the so-called criticality theory for ergodic problem (EP) (see also [3] for related results). In this paper, we investigate the asymptotic behavior of $\lambda_{\max }=\lambda_{\max }(\beta)$ as $\beta \rightarrow \infty$. More precisely, we specify the growth exponent of $\lambda_{\max }(\beta)$ as $\beta \rightarrow \infty$ in terms of constants $\delta, m$, and $\eta$ appearing in (1.1). It is known in [20, Proposition 6.2] that, if $\delta<0$ in (1.1), then $\lambda_{\max }(\beta)=0$ for all $\beta \geq 0$. It turns out in Theorem 2.3 that this is not the case for $\delta \geq 0$. Indeed, one has $\lambda_{\max }(\beta)=O\left(\beta^{\frac{m \delta}{m+\eta(m-1)}}\right)$ as $\beta \rightarrow \infty$, which particularly leads to the following asymptotic behavior:

$$
\lim _{\beta \rightarrow \infty} \lambda_{\max }(\beta)= \begin{cases}+\infty & (\delta>0) \\ \bar{\lambda} & (\delta=0) \\ 0 & (\delta<0),\end{cases}
$$

where $\bar{\lambda} \in(0, \infty)$ is some constant. To the best of our knowledge, such a classification of asymptotic behaviors of $\lambda_{\max }$ with the precision of its growth exponent has not been obtained in the literature.

From the stochastic control point of view, the previous result can be interpreted as follows. From the second part, Theorem 2.2, we observe that $\lambda_{\max }(\beta)$ is identical with the minimum value, over all admissible controls $\xi=\left(\xi_{t}\right)$, of the cost functional of long-run average

$$
J_{\beta}(x ; \xi):=\limsup _{T \rightarrow \infty} \frac{1}{T} \mathbf{E}_{x}\left[\int_{0}^{T}\left(L\left(\xi_{t}\right)+\beta V\left(X_{t}^{\xi}\right)\right) d t\right],
$$


where $L(\xi):=\left(1 / m^{*}\right)|\xi|^{m^{*}}$ with $m^{*}=m /(m-1)$ for $\xi \in \mathbf{R}^{d}$. Roughly speaking, when $\beta$ is sufficiently large, the optimal strategy for the controller is to avoid the region where $V(x)$ is large. In the case where $\delta>0$, the strength of the inward drift is such that the cost $L$ becomes dominant compared with the potential $V$. Indeed, the controller needs to compensate the drift in order to avoid the regions where $V$ is large, so that $|\xi|$, hence $L(\xi)$ is large. This implies that the cost increases as $\beta$ increases, which is also reflected on the fact that the optimal trajectory becomes positive recurrent (see Proposition 4.6). The growth order of $\lambda_{\max }(\beta)$ as $\beta \rightarrow \infty$ is, therefore, determined by the balance between the intensity $\delta$ of the inward drift $b$, the growth exponent $m^{*}$ of the cost function $L$, and the decay rate $\eta$ of the potential function $V$.

On the other hand, if $\delta=0$, then the cost incurred by $L$ is relatively small compared to that by $\beta V$, which allows the controller to avoid the peak of the potential $\beta V(x)$ without paying too much cost. As a consequence, $\lambda_{\max }(\beta)$ remains bounded uniformly in $\beta$ and converges to a finite value as $\beta \rightarrow \infty$. Let us also mention in this case that under some more assumptions we are even able to prove that $\lambda_{\max }(\beta)$ reaches a plateau, that is, there exists $\beta_{c}>0$ such that $\lambda_{\max }(\beta)=\lambda_{\max }\left(\beta_{c}\right)$ for any $\beta>\beta_{c}$ (see Proposition 6.6).

The rest of this paper is organized as follows. In the next section, we state our standing assumptions and main results precisely. Section 3 is concerned with preliminaries for later discussions. In Sections 4 and 5, we prove Theorems 2.1 and 2.2, respectively. The proof of Theorem 2.3 is given in Section 6. Appendices A, B, and C are devoted, respectively, to a gradient estimate of solutions to elliptic equations, a moment estimate of controlled processes, and a PDE characterization of value functions of finite time horizon.

2. Assumptions and Main results. For $x=\left(x_{1}, \ldots, x_{d}\right)$ and $y=\left(y_{1}, \ldots, y_{d}\right)$ in $\mathbf{R}^{d}$, we set $x \cdot y:=\sum_{i=1}^{d} x_{i} y_{i}$ and $|x|:=(x \cdot x)^{1 / 2}$. We frequently use the notation $\langle x\rangle:=\left(1+|x|^{2}\right)^{1 / 2}$ for $x \in \mathbf{R}^{d}$. Given a $d \times d$ matrix $A=\left(a_{i j}\right) \in \mathbf{R}^{d} \otimes \mathbf{R}^{d}$, we set $|A|:=\left(\sum_{i, j=1}^{d} a_{i j}^{2}\right)^{1 / 2}$. Let $B_{R}:=\left\{y \in \mathbf{R}^{d}|| y \mid<R\right\}$ and denote its closure and boundary by $\bar{B}_{R}$ and $\partial B_{R}$, respectively.

Given an integer $k \geq 0$, we denote by $C^{k}\left(\mathbf{R}^{d}\right)$ the set of all functions $u: \mathbf{R}^{d} \rightarrow \mathbf{R}$ which, together with all their partial derivatives $D^{\alpha} f$ of orders $|\alpha| \leq k$, are continuous on $\mathbf{R}^{d}$, where $\alpha=\left(\alpha_{1}, \ldots, \alpha_{d}\right)$ denotes a multi-index and $|\alpha|:=\alpha_{1}+\cdots+\alpha_{d}$. We set $C\left(\mathbf{R}^{d}\right):=C^{0}\left(\mathbf{R}^{d}\right)$ and $C^{\infty}\left(\mathbf{R}^{d}\right):=\bigcap_{k=0}^{\infty} C^{k}\left(\mathbf{R}^{d}\right)$. We also use the notation $C_{\mathrm{pol}}\left(\mathbf{R}^{d}\right)$ to denote the set of continuous functions $u: \mathbf{R}^{d} \rightarrow \mathbf{R}$ that are polynomially growing as $|x| \rightarrow \infty$, that is, $|u(x)| \leq C\langle x\rangle^{q}$ in $\mathbf{R}^{d}$ for some $C, q>0$.

We say that a sequence of functions $\left\{u_{n}\right\}$ converges to $u$ in $C^{k}\left(\mathbf{R}^{d}\right)$ as $n \rightarrow \infty$ if, for any compact subset $K \subset \mathbf{R}^{d}$ and any multi-index $\alpha$ with $|\alpha| \leq k, \max _{x \in K}\left|D^{\alpha} u_{n}(x)-D^{\alpha} u(x)\right| \rightarrow 0$ as $n \rightarrow \infty$. In particular, $\left\{u_{n}\right\}$ converges to $u$ in $C\left(\mathbf{R}^{d}\right)$ as $n \rightarrow \infty$ if and only if $\left\{u_{n}\right\}$ converges to $u$ as $n \rightarrow \infty$ uniformly on any compact subset of $\mathbf{R}^{d}$. A subset $G \subset C^{k}\left(\mathbf{R}^{d}\right)$ is said to be precompact in $C^{k}\left(\mathbf{R}^{d}\right)$ if any sequence $\left\{u_{n}\right\} \subset G$ contains a subsequence which converges in $C^{k}\left(\mathbf{R}^{d}\right)$ to a function $u \in C^{k}\left(\mathbf{R}^{d}\right)$.

Throughout the paper, we assume that $b=\left(b_{1}, \ldots, b_{d}\right)$ and $H$ satisfy the following (A1) and (A2), respectively:

(A1) $b_{i} \in C^{2}\left(\mathbf{R}^{d}\right)$ for all $1 \leq i \leq d$, and there exists a $0 \leq \delta \leq 1$ such that

$$
\sup _{x \in \mathbf{R}^{d}} \frac{|b(x)|}{\langle x\rangle^{\delta}}<\infty, \quad \sup _{x \in \mathbf{R}^{d}}|D b(x)|<\infty, \quad \liminf _{|x| \rightarrow \infty} \frac{b(x) \cdot x}{\langle x\rangle^{1+\delta}}>0,
$$

where $D b=\left(\partial b_{i} / \partial x_{j}\right): \mathbf{R}^{d} \rightarrow \mathbf{R}^{d} \otimes \mathbf{R}^{d}$.

(A2) There exist $m>1$ and $\Sigma=\left(\Sigma_{i j}(x)\right): \mathbf{R}^{d} \rightarrow \mathbf{R}^{d} \otimes \mathbf{R}^{d}$ with $\Sigma_{i j} \in C^{2}\left(\mathbf{R}^{d}\right)$ for all $i, j \in$ $\{1, \ldots, d\}$ such that

$$
H(x, p)=\frac{1}{m}\left|\Sigma^{T}(x) p\right|^{m}, \quad \inf _{x, \zeta \in \mathbf{R}^{d}, \zeta \neq 0} \frac{\left|\Sigma^{T}(x) \zeta\right|}{|\zeta|}>0, \quad \sup _{x, \zeta \in \mathbf{R}^{d}, \zeta \neq 0} \frac{\left|\Sigma^{T}(x) \zeta\right|}{|\zeta|}<\infty,
$$


where $\Sigma^{T}(x)$ denotes the transposed matrix of $\Sigma(x)$.

Note that, under condition (A2), one can easily see that

$$
\left|D_{p} H(x, p)\right| \leq K|p|^{m-1}, \quad x, p \in \mathbf{R}^{d},
$$

for some $K>0$, where $D_{p} H(x, p)$ denotes the gradient of $H(x, p)$ with respect to $p$.

As to the assumption on $V$, we consider three types of conditions:

(A3a) $V \in C^{2}\left(\mathbf{R}^{d}\right), \sup _{\mathbf{R}^{d}}|V|<\infty$, and $\sup _{\mathbf{R}^{d}}|D V|<\infty$.

(A3b) $V \in C^{2}\left(\mathbf{R}^{d}\right), \sup _{\mathbf{R}^{d}}|D V|<\infty, V \geq 0$ in $\mathbf{R}^{d}$ with $V \not \equiv 0$, and $\lim _{|x| \rightarrow \infty} V(x)=0$.

(A3c) $V \in C^{2}\left(\mathbf{R}^{d}\right), \sup _{\mathbf{R}^{d}}|D V|<\infty$, and there exists an $\eta>0$ such that

$$
\inf _{x \in \mathbf{R}^{d}}\left(V(x)\langle x\rangle^{\eta}\right)>0, \quad \sup _{x \in \mathbf{R}^{d}}\left(V(x)\langle x\rangle^{\eta}\right)<\infty .
$$

Clearly, (A3c) implies (A3b), and (A3b) yields (A3a). Hereafter, $\delta, m$, and $\eta$ always stand for the constants appearing in (A1), (A2), and (A3c), respectively. As a typical example of $(b, H, V)$ satisfying (A1), (A2), and (A3c), we have in mind functions given in (1.1).

We are in position to state our first main result. We set

$$
\begin{aligned}
& \Phi_{0}:=\left\{\phi \in C^{3}\left(\mathbf{R}^{d}\right) \mid \limsup _{|x| \rightarrow \infty} \frac{\phi(x)}{\langle x\rangle^{1+\frac{\delta}{m-1}}}<\infty, \quad \liminf _{|x| \rightarrow \infty} \frac{\phi(x)}{\left.\langle x\rangle^{\gamma} \geq 0, \quad \forall \gamma>1-\delta\right\},}\right. \\
& \Phi_{1}:=\left\{\phi \in C^{3}\left(\mathbf{R}^{d}\right) \mid \limsup _{|x| \rightarrow \infty} \frac{\phi(x)}{\langle x\rangle^{1-\delta}}<0, \quad \liminf _{|x| \rightarrow \infty} \frac{\phi(x)}{\langle x\rangle^{\gamma}} \geq 0, \quad \forall \gamma>1-\delta\right\} .
\end{aligned}
$$

Note that $\Phi_{1} \subset \Phi_{0}$. Roughly speaking, functions in $\Phi_{1}$ look like $-c\langle x\rangle^{1-\delta}(1+o(1))$ for some $c>0$ as $|x| \rightarrow \infty$. In particular, any function in $\Phi_{1}$ is bounded above in $\mathbf{R}^{d}$. The class $\Phi_{0}$ contains functions $\phi$ such that $-C\langle x\rangle^{1-\delta} \leq \phi(x) \leq C\langle x\rangle^{1+\frac{\delta}{m-1}}$ in $\mathbf{R}^{d}$ for some $C>0$.

Let $\lambda_{\max }$ be the generalized principal eigenvalue of (EP) defined by (1.2). Note that $\lambda_{\max }>$ $-\infty$ since $(\lambda, \phi):=\left(-\sup _{\mathbf{R}^{d}}|V|, 0\right)$ is a subsolution of (EP) under (A1), (A2), and (A3a). Then one has the following characterization of solutions to $(\mathrm{EP})$.

Theorem 2.1. Assume (A1) with $\delta>0$, (A2), and (A3a). Then the following (a)-(c) hold.

(a) A solution $(\lambda, \phi) \in \mathbf{R} \times C^{2}\left(\mathbf{R}^{d}\right)$ of (EP) satisfies $\phi \in \Phi_{0}$ if and only if $\lambda=\lambda_{\max }$.

(b) There exists a unique solution $\left(\lambda^{*}, \phi^{*}\right) \in \mathbf{R} \times C^{3}\left(\mathbf{R}^{d}\right)$ of (EP), up to an additive constant with respect to $\phi^{*}$, such that $\phi^{*} \in \Phi_{0}$. In particular, $\lambda^{*}=\lambda_{\max }$, and $\phi^{*}$ is the unique eigenfunction of (EP) associated with $\lambda_{\max }$, up to an additive constant.

(c) If we assume (A3b) instead of (A3a), then $\phi^{*} \in \Phi_{1}$.

In order to state our second result, we formulate the stochastic control problem associated with (EP). We say that the 6-tuple

$$
\pi=\left(\Omega, \mathcal{F},\left(\mathcal{F}_{t}\right)_{t \geq 0}, P ;\left(W_{t}\right)_{t \geq 0} ;\left(\xi_{t}\right)_{t \geq 0}\right)
$$

is an admissible control if $(\Omega, \mathcal{F}, P)$ is a complete probability space, $\left(\mathcal{F}_{t}\right)_{t \geq 0}$ a family of sub- $\sigma$ algebras satisfying $\mathcal{N} \subset \mathcal{F}_{t} \subset \mathcal{F}_{s}$ and $\mathcal{F}_{t}=\bigcap_{u>t} \mathcal{F}_{u}$ for all $0 \leq t \leq s$, where $\mathcal{N}$ is the collection of all $P$-null sets, $\left(W_{t}\right)_{t \geq 0}$ a $d$-dimensional $\left(\mathcal{F}_{t}\right)$-Brownian motion, and $\xi=\left(\xi_{t}\right)_{t \geq 0}$ an $\mathbf{R}^{d}$-valued $\left(\mathcal{F}_{t}\right)$-progressively measurable process such that

$$
\mathbf{E}\left[\int_{0}^{T}\left|\xi_{t}\right|^{m^{*}} d t\right]<\infty \text { for all } T>0,
$$

where $m^{*}:=m /(m-1)$. Let $\mathcal{A}$ denote the collection of all admissible controls $\pi$. As usual, we use the conventional notation $\xi \in \mathcal{A}$, instead of $\pi \in \mathcal{A}$. 
Given an admissible control $\xi \in \mathcal{A}$, we denote by $X^{\xi}=\left(X_{t}^{\xi}\right)_{t \geq 0}$ the associated controlled process governed by (1.3). It is known (e.g. [30, Section 1]) that, for any initial point $x \in \mathbf{R}^{d}$, there exists a unique solution of (1.3) which does not explode in finite time.

We next introduce the cost functional to be optimized which is slightly general than (1.4). Let $g: \mathbf{R}^{d} \rightarrow \mathbf{R}$ be a given terminal cost belonging to the class $\Psi_{\alpha}$ with $\alpha=1+\frac{\delta}{m-1}$, where

$$
\Psi_{\alpha}:=\left\{g \in C_{\mathrm{pol}}\left(\mathbf{R}^{d}\right) \mid \liminf _{|x| \rightarrow \infty} \frac{g(x)}{\langle x\rangle^{\alpha}} \geq 0\right\}, \quad \alpha \geq 0 .
$$

For $T>0, x \in \mathbf{R}^{d}$, and $\xi \in \mathcal{A}$, we set

$$
J(T, x ; \xi):=\mathbf{E}_{x}\left[\int_{0}^{T}\left(L\left(X_{t}^{\xi}, \xi_{t}\right)+V\left(X_{t}^{\xi}\right)\right) d t+g\left(X_{T}^{\xi}\right)\right],
$$

where $L: \mathbf{R}^{d} \times \mathbf{R}^{d} \rightarrow \mathbf{R}$ is the convex conjugate of $H$ defined by

$$
L(x, \xi):=\sup _{p \in \mathbf{R}^{d}}(\xi \cdot p-H(x, p)), \quad x, \xi \in \mathbf{R}^{d} .
$$

Note that, if we denote by $\Sigma^{-1}(x)$ the inverse matrix of $\Sigma(x)$ appearing in (A2), then $L(x, \xi)$ can be explicitly written as

$$
L(x, \xi):=\frac{1}{m^{*}}\left|\Sigma^{-1}(x) \xi\right|^{m^{*}}, \quad x, \xi \in \mathbf{R}^{d} .
$$

One can also verify from $(2.6)$ that $H(x, p)+L(x, \xi) \geq \xi \cdot p$ for all $x, p, \xi \in \mathbf{R}^{d}$, and that $H(x, p)+$ $L(x, \xi)=\xi \cdot p$ if and only if $\xi=D_{p} H(x, p)$.

We finally define the value functions of finite time horizon $T$ and of long-run average by

$$
u(T, x):=\inf _{\xi \in \mathcal{A}} J(T, x ; \xi), \quad \Lambda(x):=\inf _{\xi \in \mathcal{A}} \limsup _{T \rightarrow \infty} \frac{J(T, x ; \xi)}{T},
$$

respectively. Then, our second result can be stated as follows.

Theorem 2.2. Assume (A1) with $\delta>0$, (A2), (A3b), and one of the following (i)-(ii):

(i) $g \in \Psi_{1-\delta}$;

(ii) $g \in \Psi_{m^{*}}$ and $\delta=1$ in (A1).

Then, $\Lambda(x)=\lambda_{\max }$ for all $x \in \mathbf{R}^{d}$. Moreover, there exists a constant $c \in \mathbf{R}$ such that

$$
u(T, x)-\lambda_{\max } T \longrightarrow \phi^{*}(x)+c \quad \text { in } C\left(\mathbf{R}^{d}\right) \text { as } T \rightarrow \infty,
$$

where $\phi^{*}$ is the eigenfunction of (EP) associated with $\lambda_{\max }$, given in Theorem 2.1.

Now, we state our third result. Let us introduce the real parameter $\beta \geq 0$ and consider ergodic problem (EP) with $\beta V$ instead of $V$, that is,

$$
\lambda-\frac{1}{2} \Delta \phi+b(x) \cdot D \phi+H(x, D \phi)-\beta V=0 \text { in } \mathbf{R}^{d} .
$$

Let $\lambda_{\max }=\lambda_{\max }(\beta)$ denote the generalized principal eigenvalue of $\left(\mathrm{EP}_{\beta}\right)$ defined similarly as (1.2). Then $\lambda_{\max }(\beta)$ has the following properties.

TheOREm 2.3. Assume (A1), (A2), and (A3c). Then $\lambda_{\max }(0)=0$, and $\lambda_{\max }(\beta)$ is strictly positive, nondecreasing, and concave in $\beta \in(0, \infty)$. Moreover, there exists some $\nu>0$ such that

$$
\nu \beta^{\frac{m \delta}{m \delta+\eta(m-1)}}-\nu^{-1} \leq \lambda_{\max }(\beta) \leq \nu^{-1}\left(1+\beta^{\frac{m \delta}{m \delta+\eta(m-1)}}\right), \quad \beta \geq 0 .
$$

In particular, as $\beta \rightarrow \infty, \lambda_{\max }(\beta)$ diverges to $+\infty$ for $\delta>0$, while $\lambda_{\max }(\beta)$ converges to a finite value $\bar{\lambda} \in(0, \infty)$ for $\delta=0$. 
In view of Theorem 2.3, in the case where $\delta>0$, since $\beta \mapsto \lambda_{\max (\beta)}$ is concave and diverges to $\infty$ as $\beta \rightarrow \infty$, we deduce that $\lambda_{\max }(\beta)$ is strictly increasing with respect to $\beta$. We will see in Section 6 that this is not the case, in general, for $\delta=0$. Indeed, under some additional assumptions on $(b, H, V)$, it happens that there exists a $\beta_{c}>0$ such that $\lambda_{\max }(\beta)=\lambda_{\max }\left(\beta_{c}\right)$ for all $\beta \geq \beta_{c}$. This kind of "flattening" phenomena have been observed and discussed by $[19,20]$ in connection with the criticality theory for ergodic problems. See $[19,20]$ for details.

We close this section with some remarks on the extension of Theorems 2.1 and 2.2 to the case where $\delta=0$. In that case, it is also possible to obtain similar results under certain additional assumptions. For instance, under the hypothesis of Theorem 2.3 with $\delta=0$, Theorems 2.1 and 2.2 still hold provided $\lambda_{\max }(\beta)<\bar{\lambda}:=\sup \left\{\lambda_{\max }\left(\beta^{\prime}\right) \mid \beta^{\prime} \geq 0\right\}$. This is a direct consequence of [20, Theorem 2.2] for Theorem 2.1 and of [20, Theorem 2.1] for the first half of Theorem 2.2, i.e., for the validity of $\Lambda(x)=\lambda_{\max }$. For the second half of Theorem 2.2, one can apply the same argument developed in Section 5 of this paper to establish the convergence (2.9). In any case, it is crucial to assume the condition $\lambda_{\max }(\beta)<\bar{\lambda}$, which is the key to the ergodicity of the optimal trajectory as well as the uniqueness of eigenfunctions associated with the generalized principal eigenvalue (see Section 4). As is mentioned, this condition is trivial if $\delta>0$ since $\bar{\lambda}=\infty$, whereas, if $\delta=0$, it may occur that $\lambda_{\max }(\beta)=\bar{\lambda}$ for some $\beta$, and that the optimal trajectory becomes transient (cf. $[19,20])$. We refer to $[3]$ for related results in this direction.

3. Preliminaries. In this section, we always assume (A1) and (A2). For a given $\phi \in C^{2}\left(\mathbf{R}^{d}\right)$, we define the second order partial differential operator $A^{\phi}$ by

$$
A^{\phi}:=\frac{1}{2} \Delta-b(x) \cdot D-D_{p} H(x, D \phi(x)) \cdot D,
$$

where $D=\left(\partial / \partial x_{1}, \cdots, \partial / \partial x_{d}\right)$ and $\Delta:=\sum_{j=1}^{d} \partial^{2} / \partial x_{j}^{2}$. We consider the stochastic differential equation

$$
\left\{\begin{array}{l}
d X_{t}=-D_{p} H\left(X_{t}, D \phi\left(X_{t}\right)\right) d t-b\left(X_{t}\right) d t+d W_{t}, \quad t \geq 0 \\
X_{0}=x
\end{array}\right.
$$

It is well known (see, for instance, [30, Theorem 11.1]) that, for any $\phi \in C^{2}\left(\mathbf{R}^{d}\right)$, there exists a filtered probability space $\left(\Omega, \mathcal{F},\left(\mathcal{F}_{t}\right)_{t \geq 0}, P\right)$ and an $\left(\mathcal{F}_{t}\right)$-Brownian motion $W=\left(W_{t}\right)_{t \geq 0}$ such that (3.1) has a unique solution up to its explosion time $\tau_{\infty}:=\lim _{n \rightarrow \infty} \tau_{n}$, where $\tau_{n}:=\inf \left\{t>0|| X_{t} \mid>n\right\}$ for $n \geq 1$. In this paper, we shall call the solution of (3.1) the $A^{\phi}$-diffusion. The rest of this section is devoted to recalling some fundamental results on $A^{\phi}$-diffusions that are well known in the literature not only for $A^{\phi}$-diffusions but also for more general diffusion processes in $\mathbf{R}^{d}$.

We begin with the following fact that will be used in later discussions.

Proposition 3.1. Let $X=\left(X_{t}\right)_{t \geq 0}$ be the $A^{\phi}$-diffusion for some $\phi \in C^{2}\left(\mathbf{R}^{d}\right)$.

(a) Suppose that $X$ is positive recurrent. Let $\mu=\mu(d y)$ be its invariant probability measure on $\mathbf{R}^{d}$. Then, for any measurable $f: \mathbf{R}^{d} \rightarrow \mathbf{R}$ such that $\int_{\mathbf{R}^{d}}|f(y)| \mu(d y)<\infty$,

$$
\mathbf{E}_{x}\left[f\left(X_{T}\right)\right] \longrightarrow \int_{\mathbf{R}^{d}} f(y) \mu(d y) \text { as } T \rightarrow \infty,
$$

where the convergence is uniform, with respect to $x$, on any compact subset of $\mathbf{R}^{d}$.

(b) Suppose that $X$ is not positive recurrent. Then, for any $f: \mathbf{R}^{d} \rightarrow \mathbf{R}$ with compact support, and for any $x \in \mathbf{R}^{d}$,

$$
\mathbf{E}_{x}\left[f\left(X_{T}\right)\right] \longrightarrow 0 \quad \text { as } T \rightarrow \infty
$$

Proof. Claim (a) has been proved in [21, Proposition 2.6]. For the proof of (b), see, for instance, $[24$, Theorem 1.3.10]. 
The recurrence and transience of $A^{\phi}$-diffusions can be verified by using the following proposition, which we call the Lyapunov method in this paper.

Proposition 3.2. Let $X=\left(X_{t}\right)_{t \geq 0}$ be the $A^{\phi}$-diffusion for some $\phi \in C^{2}\left(\mathbf{R}^{d}\right)$.

(a) Suppose that there exist $R>0, u \in C^{2}\left(\mathbf{R}^{d} \backslash B_{R}\right)$, and $K>0$ such that

$$
\lim _{|x| \rightarrow \infty} u(x)=\infty, \quad A^{\phi} u \leq K u \quad \text { in } \mathbf{R}^{d} \backslash B_{R} .
$$

Then $X$ does not explode in finite time, i.e., $\mathbf{P}_{x}\left(\tau_{\infty}=\infty\right)=1$ for all $x \in \mathbf{R}^{d}$.

(b) Suppose that there exist $R>0, u \in C^{2}\left(\mathbf{R}^{d} \backslash B_{R}\right)$, and $x_{0} \notin \bar{B}_{R}$ such that

$$
\inf _{\mathbf{R}^{d} \backslash B_{R}} u>-\infty, \quad u\left(x_{0}\right)<\min _{\partial B_{R}} u, \quad A^{\phi} u \leq 0 \quad \text { in } \mathbf{R}^{d} \backslash B_{R} .
$$

Then $X$ is transient.

(c) Suppose that there exist $R>0$ and $u \in C^{2}\left(\mathbf{R}^{d} \backslash B_{R}\right)$ such that

$$
\lim _{|x| \rightarrow \infty} u(x)=\infty, \quad A^{\phi} u \leq 0 \quad \text { in } \mathbf{R}^{d} \backslash B_{R} .
$$

Then $X$ is recurrent.

(d) Suppose that $X$ does not explode in finite time, and that there exist $R>0, u \in C^{2}\left(\mathbf{R}^{d} \backslash B_{R}\right)$, and $\varepsilon>0$ such that

$$
\inf _{\mathbf{R}^{d} \backslash B_{R}} u>-\infty, \quad A^{\phi} u \leq-\varepsilon \quad \text { in } \mathbf{R}^{d} \backslash B_{R} .
$$

Then $X$ is positive recurrent with an invariant probability measure $\mu=\mu(d y)$ which has a positive and continuous density in $\mathbf{R}^{d}$. More generally, suppose that there exist some $u \in C^{2}\left(\mathbf{R}^{d}\right), f \in$ $C\left(\mathbf{R}^{d}\right), K>0$, and $R>0$ such that

$$
\inf _{\mathbf{R}^{d}} u>-\infty, \quad \inf _{\mathbf{R}^{d} \backslash B_{R}} f>0, \quad A^{\phi} u \leq-f \mathbf{1}_{\mathbf{R}^{d} \backslash B_{R}}+K \mathbf{1}_{B_{R}} \quad \text { in } \mathbf{R}^{d} .
$$

Then the invariant probability measure $\mu$ of $X$ satisfies $\int_{\mathbf{R}^{d}} f(y) \mu(d y)<\infty$.

Proof. We refer to [30, Theorems 6.7.1, 6.1.1, 6.1.2] for the proofs of (a)-(c). The positive recurrence of $X$ stated in (d), as well as the existence of a positive continuous density of $\mu$ are proved in [30, Theorem 6.1.3] and [30, Section 4.8], respectively (see, especially, Theorem 4.8.4 for the latter). Here, we only check the integrability of $f$ with respect to $\mu$, which is also classical but is not explicitly mentioned in [30].

We may assume without loss of generality that $u \geq 0$ in $\mathbf{R}^{d}$. Applying Ito's formula to $u\left(X_{t}\right)$, we have

$$
\begin{aligned}
\mathbf{E}_{x}\left[u\left(X_{T \wedge \tau_{n}}\right)\right]-u(x) & =\mathbf{E}_{x}\left[\int_{0}^{T \wedge \tau_{n}} A^{\phi} u\left(X_{t}\right) d t\right] \\
& \leq \mathbf{E}_{x}\left[\int_{0}^{T \wedge \tau_{n}}\left(-f\left(X_{t}\right) \mathbf{1}_{\mathbf{R}^{d} \backslash B_{R}}\left(X_{t}\right)+K \mathbf{1}_{B_{R}}\left(X_{t}\right)\right) d t\right]
\end{aligned}
$$

for all $x \in \mathbf{R}^{d}, T>0$, and $n \geq 1$, where $\tau_{n}:=\inf \left\{t>0|| X_{t} \mid>n\right\}$. Since $u$ and $f$ are bounded below in $\mathbf{R}^{d}$ and $X$ does not explode in finite time, we see by letting $n \rightarrow \infty$ that

$$
\mathbf{E}_{x}\left[\int_{0}^{T} f\left(X_{t}\right) \mathbf{1}_{\mathbf{R}^{d} \backslash B_{R}}\left(X_{t}\right) d t\right] \leq u(x)+K T .
$$

Dividing both sides by $T$ and then sending $T \rightarrow \infty$, we observe in view of the ergodic theorem for the $A^{\phi}$-diffusion that

$$
\int_{\mathbf{R}^{d} \backslash B_{R}} f(y) \mu(d y) \leq \liminf _{T \rightarrow \infty} \frac{1}{T} \mathbf{E}_{x}\left[\int_{0}^{T} f\left(X_{t}\right) \mathbf{1}_{\mathbf{R}^{d} \backslash B_{R}}\left(X_{t}\right) d t\right] \leq K,
$$


which implies the desired estimate. Hence, we have completed the proof of (d).

REMARK 3.3. In view of (3.2) and Fatou's lemma, we observe that

$$
\sup _{n \geq 1} \mathbf{E}_{x}\left[u\left(X_{T \wedge \tau_{n}}\right)\right] \leq u(x)+K T, \quad \mathbf{E}_{x}\left[u\left(X_{T}\right)\right] \leq u(x)+K T
$$

for all $x \in \mathbf{R}^{d}$ and $T>0$. These estimates will be used in the proof of Proposition 4.6.

Hereafter, for the simplicity of notation, we set

$$
F[\phi](x):=-\frac{1}{2} \Delta \phi(x)+b(x) \cdot D \phi(x)+H(x, D \phi(x)), \quad x \in \mathbf{R}^{d}, \quad \phi \in C^{2}\left(\mathbf{R}^{d}\right) .
$$

The next proposition is the key to the construction of Lyapunov functions $u$ appearing in Proposition 3.2.

Proposition 3.4. Let $D \subset \mathbf{R}^{d}$ be a domain, and let $\phi, \psi \in C^{2}(D)$.

(a) $u:=\phi-\psi$ satisfies $A^{\phi} u \leq F[\psi]-F[\phi]$ in $D$.

(b) Suppose that $m \geq 2$ in (A2). Then, for any $\varepsilon>0$, there exists a $\kappa>0$ such that $u:=e^{\kappa(\phi-\psi)}$ satisfies

$$
A^{\phi} u \leq \kappa u(F[\psi]-F[\phi]+\varepsilon) \text { in } D .
$$

Moreover, if $m=2$ in (A2), then the above inequality holds with $\varepsilon=0$.

(c) Suppose that $1<m<2$ in (A2). Then, for any $K>0$, there exists a $\kappa>0$ such that, for any $\phi, \psi$ with $|D \phi|+|D \psi| \leq K$ in $D$, the function $u:=e^{\kappa(\phi-\psi)}$ satisfies

$$
A^{\phi} u \leq \kappa u(F[\psi]-F[\phi]) \text { in } D \text {. }
$$

Proof. We reproduce a sketch of the proof for the convenience of the reader (see [20, Proposition 3.10] for a complete proof). Claim (a) is a direct consequence of the convexity of $p \mapsto H(x, p)$. Indeed, noting that $H(x, q)-H(x, p) \geq D_{p} H(x, p) \cdot(q-p)$, we see that $u:=\phi-\psi$ satisfies

$$
\begin{aligned}
A^{\phi} u & =A \phi-A \psi-D_{p} H(x, D \phi) \cdot(D \phi-D \psi) \\
& \leq A \phi-H(x, D \phi)-(A \psi-H(x, D \psi))=F[\psi]-F[\phi],
\end{aligned}
$$

where $A:=(1 / 2) \Delta-b(x) \cdot D$. Hence, (a) is valid. We next prove (b). Fix any $m \geq 2$ and $\varepsilon>0$. Then we observe from (A2) that

$$
H(x, p+q)-H(x, p)-D_{p} H(x, p) \cdot q \geq \frac{\kappa}{2}|q|^{2}-\varepsilon, \quad x, p, q \in \mathbf{R}^{d},
$$

for some $\kappa>0$ (see, e.g., [20, Proposition 3.7]). Using this inequality, we see by direct computations similar to (a) that $u:=e^{\kappa w}$ with $w:=\phi-\psi$ satisfies

$$
\begin{aligned}
A^{\phi} u & =\kappa u\left(A^{\phi} w+\frac{\kappa}{2}|D w|^{2}\right) \leq \kappa u\left(F[\psi]-F[\phi]-\frac{\kappa}{2}|D w|^{2}+\varepsilon+\frac{\kappa}{2}|D w|^{2}\right) \\
& =\kappa u(F[\psi]-F[\phi]+\varepsilon) .
\end{aligned}
$$

If $m=2$ in (A2), then (3.3) holds with $\varepsilon=0$. This leads to the inequality $A^{\phi} u \leq \kappa u(F[\psi]-F[\phi])$ in $D$. Hence, (b) is valid. We finally verify (c). Fix any $1<m<2$ and $K>0$. Then, we observe that

$$
H(x, p+q)-H(x, p)-D_{p} H(x, p) \cdot q \geq \frac{\kappa}{2}|q|^{2}, \quad x \in \mathbf{R}^{d}, \quad|p|,|q| \leq K,
$$

for some $\kappa>0$ (see, e.g., [20, Proposition 3.7]). By the same argument as (b), we conclude that $u:=e^{\kappa w}$ with $w:=\phi-\psi$ satisfies

$$
A^{\phi} u=\kappa u\left(A^{\phi} w+\frac{\kappa}{2}|D w|^{2}\right) \leq \kappa u(F[\psi]-F[\phi]) .
$$

Hence, we have completed the proof. 
4. Proof of Theorem 2.1. This section is devoted to the proof of Theorem 2.1. Unless otherwise specified, we assume (A1) with $\delta>0,(\mathrm{~A} 2)$, and (A3a). Note that, for any solution $(\lambda, \phi) \in \mathbf{R} \times C^{2}\left(\mathbf{R}^{d}\right)$ of (EP), $\phi$ is indeed of $C^{3}$-class. This is a direct consequence of classical Schauder type estimates for linear elliptic equations (e.g. [15, Theorem 6.17]). Hence, as far as solutions $(\lambda, \phi) \in \mathbf{R} \times C^{2}\left(\mathbf{R}^{d}\right)$ of (EP) are concerned, we may assume without loss of generality that $\phi \in C^{3}\left(\mathbf{R}^{d}\right)$.

We begin with an a priori gradient estimate for eigenfunctions $\phi$ of (EP) which plays a crucial role throughout the paper.

Proposition 4.1. There exists a constant $K>0$ such that, for any solution $(\lambda, \phi) \in \mathbf{R} \times$ $C^{3}\left(\mathbf{R}^{d}\right)$ of $(E P)$,

$$
|D \phi(x)| \leq K\left(1+|x|^{\frac{\delta}{m-1}}+\left(\lambda_{-}\right)^{\frac{1}{m}}\right), \quad x \in \mathbf{R}^{d},
$$

where $r_{ \pm}:=\max \{ \pm r, 0\}$ for $r \in \mathbf{R}$. In particular,

$$
\sup _{x \in \mathbf{R}^{d}} \frac{|\phi(x)|}{\langle x\rangle^{1+\frac{\delta}{m-1}}}<\infty .
$$

Proof. Let $(\lambda, \phi) \in \mathbf{R} \times C^{3}\left(\mathbf{R}^{d}\right)$ be a solution of (EP). Then, in view of Theorem A.2 in Appendix A, there exists a $K>0$ depending only on $m$ and $d$ such that, for any $r>0$,

$$
\sup _{B_{r}}|D \phi| \leq K\left\{1+\sup _{B_{r+1}}\left(|b|^{\frac{1}{m-1}}+|D b|^{\frac{1}{2(m-1)}}+(V-\lambda)_{+}^{\frac{1}{m}}+|D V|^{\frac{1}{2 m-1}}\right)\right\} .
$$

From this estimate, together with (A1) and (A3a), we obtain the claim.

We next state an existence result for a solution of (EP).

Proposition 4.2. Let $\lambda_{\max }$ be the generalized principal eigenvalue of (EP) defined by (1.2). Then, for any $\lambda \leq \lambda_{\max }$, there exists an eigenfunction $\phi \in C^{3}\left(\mathbf{R}^{d}\right)$ of (EP) associated with $\lambda$.

Proof. We only give a sketch of the proof since the proof of this proposition is standard (cf. $[9,19,20])$. We first claim that, if $\left(\lambda_{0}, \phi_{0}\right) \in \mathbf{R} \times C^{3}\left(\mathbf{R}^{d}\right)$ is a subsolution of (EP) and $\varepsilon>0$ is arbitrary, then there exists an eigenfunction $\phi \in C^{3}\left(\mathbf{R}^{d}\right)$ of (EP) associated with $\lambda_{0}-\varepsilon$. This implies that, for any $\lambda<\lambda_{\max }$, there exists an eigenfunction of (EP) associated with $\lambda$. In order to show this claim, fix any $R>0$ and consider the Dirichlet problem

$$
\lambda_{0}-\varepsilon+F[u]-V=0 \quad \text { in } B_{R}, \quad u=\phi_{0} \quad \text { on } \partial B_{R} .
$$

Then, in view of Theorem A.1 in Appendix A, there exists a solution $u_{R} \in C^{3}\left(B_{R}\right) \cap C\left(\bar{B}_{R}\right)$ of (4.1). Furthermore, by Proposition 4.1, togerther with the standard regularity estimates (e.g. [27, Theorem 4.6.1] and [15, Theorem 4.6]), one can see that, along a suitable diverging sequence $\left\{R_{j}\right\}$, the family $\left\{u_{R_{j}}-u_{R_{j}}(0)\right\}_{j \geq 1}$ converges to a function $\phi$ in $C^{2}\left(\mathbf{R}^{N}\right)$ as $j \rightarrow \infty$, and that $\phi$ is of $C^{3}$-class and enjoys (EP) with $\lambda=\lambda_{0}-\varepsilon$. In order to construct an eigenfunction $\phi \in C^{3}\left(\mathbf{R}^{d}\right)$ of (EP) associated with $\lambda_{\max }$, we choose any increasing sequence $\left\{\lambda^{(n)}\right\}$ such that $\lambda^{(n)} \rightarrow \lambda_{\max }$ as $n \rightarrow \infty$, and let $\left\{\phi^{(n)}\right\}$ denote a sequence of associated eigenfunctions of (EP). Then, similarly as above, one can see that, along a suitable subsequence, $\left\{\phi^{(n)}-\phi^{(n)}(0)\right\}$ converges to a function $\phi$ in $C^{2}\left(\mathbf{R}^{N}\right)$ as $n \rightarrow \infty$. In particular, $\phi$ satisfies (EP) with $\lambda=\lambda_{\max }$, and therefore $\phi \in C^{3}\left(\mathbf{R}^{d}\right)$. Hence, we have completed the proof.

The above existence result does not give any useful information on the asymptotic behavior of eigenfunctions $\phi$ as $|x| \rightarrow \infty$. In what follows, we construct a special class of solution $\left(\lambda^{*}, \phi^{*}\right)$ to (EP) such that $\phi^{*} \in \Phi_{0}$. To this end, we begin with the following abstract result.

Proposition 4.3. Suppose that there exists a triplet $\left(\psi_{-1}, \psi_{0}, \psi_{1}\right)$ of $C^{3}$-functions which satisfies the following (i)-(iii):

(i) $\psi_{-1} \leq \psi_{0} \leq \psi_{1}$ in $\mathbf{R}^{d}, \quad \lim _{|x| \rightarrow \infty}\left(\psi_{0}-\psi_{-1}\right)(x)=\infty$, 
(ii) $\limsup _{|x| \rightarrow \infty}\left(\lambda_{\max }+F\left[\psi_{-1}\right]-V\right)(x)<0, \quad \limsup _{|x| \rightarrow \infty}\left(\lambda_{\max }+F\left[\psi_{0}\right]-V\right)(x)<0$,

(iii) $\inf _{\mathbf{R}^{d}} F\left[\psi_{1}\right]>-\infty$.

Then, there exists a solution $\left(\lambda^{*}, \phi^{*}\right)$ of $(E P)$ such that $\inf _{\mathbf{R}^{d}}\left(\phi^{*}-\psi_{0}\right)>-\infty$, and the associated $A^{\phi^{*}}$-diffusion is positive recurrent. Moreover, if $\psi_{1}$ satisfies

$(\text { iii) })^{\prime} \liminf _{|x| \rightarrow \infty}\left(\lambda^{*}+F\left[\psi_{1}\right]-V\right)(x)>0$,

then $\inf _{\mathbf{R}^{d}}\left(\psi_{1}-\phi^{*}\right)>-\infty$.

Proof. Fix any $R>0$ and consider the following Dirichlet problem with discount factor $\alpha>0$ :

$$
\alpha v+F[v]-V=\alpha \psi_{0} \text { in } B_{R}, \quad v=\psi_{0} \quad \text { on } \partial B_{R}
$$

Since $F\left[\psi_{0}\right] \leq K$ and $F\left[\psi_{1}\right] \geq-K$ in $\mathbf{R}^{d}$ for some $K>0$, we observe, by setting $C:=K+$ $\sup _{\mathbf{R}^{d}}|V|$, that $\psi_{0}-\alpha^{-1} C$ and $\psi_{1}+\alpha^{-1} C$ are, respectively, a subsolution and a supersolution of (4.2). In particular, by virtue of Theorem A.1 in Appendix A, there exists a solution $v_{\alpha, R} \in$ $C^{3}\left(B_{R}\right) \cap C\left(\bar{B}_{R}\right)$ of (4.2) such that $\psi_{0}-\alpha^{-1} C \leq v_{\alpha, R} \leq \psi_{1}+\alpha^{-1} C$ in $\bar{B}_{R}$. Noting that $\left\{v_{\alpha, R}\right\}_{R>0}$ is precompact in $C^{2}\left(\mathbf{R}^{d}\right)$ in view of classical regularity estimates for elliptic equations, one can find a function $v_{\alpha} \in C^{3}\left(\mathbf{R}^{d}\right)$ which satisfies $\psi_{0}-\alpha^{-1} C \leq v_{\alpha} \leq \psi_{1}+\alpha^{-1} C$ in $\mathbf{R}^{d}$ and

$$
\alpha v+F[v]-V=\alpha \psi_{0} \quad \text { in } \mathbf{R}^{d} .
$$

Let $\left\{\alpha_{n}\right\}$ be any positive decreasing sequence such that $\alpha_{n} \rightarrow 0$ as $n \rightarrow \infty$, and set $\lambda_{n}:=$ $\alpha_{n} v_{\alpha_{n}}(0)$ and $w_{n}:=v_{\alpha_{n}}-v_{\alpha_{n}}(0)$. Then, $\left(\lambda_{n}, w_{n}\right)$ satisfies

$$
\lambda_{n}+\alpha_{n} w_{n}+F\left[w_{n}\right]-V=\alpha_{n} \psi_{0} \quad \text { in } \mathbf{R}^{d} .
$$

Since $\alpha_{n} \psi_{0}(0)-C \leq \lambda_{n} \leq \alpha_{n} \psi_{1}(0)+C$ in $\mathbf{R}^{d}$, we see that $\left\{\lambda_{n}\right\}$ is bounded in $\mathbf{R}$. Furthermore, by Theorem A.2 in Appendix A, we observe that $\sup _{n \geq 1} \sup _{B_{R}}\left|D w_{n}\right|<\infty$ for all $R>0$. This and the standard Schauder estimates imply that $\left\{w_{n}\right\}$ is precompact in $C^{2}\left(\mathbf{R}^{d}\right)$. Thus, there exists a subsequence of $\left\{\alpha_{n}\right\}$, denoted again by $\left\{\alpha_{n}\right\}$, and a pair $\left(\lambda^{*}, \phi^{*}\right) \in \mathbf{R} \times C^{2}\left(\mathbf{R}^{d}\right)$ such that $\lambda_{n} \rightarrow \lambda^{*}$ as $n \rightarrow \infty$ and $w_{n} \rightarrow \phi^{*}$ in $C^{2}\left(\mathbf{R}^{d}\right)$ as $n \rightarrow \infty$. Letting $n \rightarrow \infty$ in (4.4), we conclude that $\left(\lambda^{*}, \phi^{*}\right)$ is a solution of (EP) and $\phi^{*} \in C^{3}\left(\mathbf{R}^{d}\right)$.

Next, we verify that the above $\left(\lambda^{*}, \phi^{*}\right)$ is the desired solution. In view of assumption (ii), we observe that there exist some $\rho>0$ and $R>0$ such that, for any $x \in \mathbf{R}^{d} \backslash B_{R}$,

$$
\lambda_{\max }+F\left[\psi_{-1}\right](x)-V(x) \leq-\rho, \quad \lambda_{\max }+F\left[\psi_{0}\right](x)-V(x) \leq-\rho .
$$

Since $\lambda_{n} \rightarrow \lambda^{*}$ as $n \rightarrow \infty$, we may assume without loss of generality that $\left|\lambda_{n}-\lambda^{*}\right|<\rho$ for all $n \geq 1$. Then, noting that $\lambda^{*} \leq \lambda_{\max }$, we obtain

$$
\lambda_{n}+F\left[\psi_{i}\right](x)-V(x)<0, \quad x \in \mathbf{R}^{d} \backslash B_{R}, \quad n \geq 1, \quad i=-1,0 .
$$

We now set $\psi_{-\theta}:=(1-\theta) \psi_{0}+\theta \psi_{-1}$ for $\theta \in(0,1)$, and

$$
M:=\sup _{B_{R}}\left(\sup _{n \geq 1}\left|w_{n}\right|+\left|\psi_{-1}\right|+\left|\psi_{0}\right|+\left|\psi_{1}\right|\right)<\infty .
$$

We show that $\psi_{-\theta}-M \leq w_{n}$ in $\mathbf{R}^{d}$ for any $\theta \in(0,1)$ and $n \geq 1$. From the definition of $M$, it is clear that $\psi_{-\theta}-M \leq w_{n}$ in $\bar{B}_{R}$. One can also see that, as $|x| \rightarrow \infty$,

$$
\begin{aligned}
w_{n}-\psi_{-\theta} & =w_{n}-\psi_{0}+\theta\left(\psi_{0}-\psi_{-1}\right)=\left(v_{\alpha_{n}}-\psi_{0}\right)-v_{\alpha_{n}}(0)+\theta\left(\psi_{0}-\psi_{-1}\right) \\
& \geq-\frac{C}{\alpha_{n}}-v_{\alpha_{n}}(0)+\theta\left(\psi_{0}-\psi_{-1}\right) \geq-\psi_{1}(0)-\frac{2 C}{\alpha_{n}}+\theta\left(\psi_{0}-\psi_{-1}\right) \rightarrow \infty
\end{aligned}
$$

In particular, for each $n \geq 1$, there exists an $R_{n}>R$ such that $\psi_{-\theta}-M \leq w_{n}$ in $\mathbf{R}^{d} \backslash B_{R_{n}}$. 
Set $D_{n}:=B_{R_{n}} \backslash \bar{B}_{R}$. Then, since $F\left[\psi_{-\theta}\right] \leq(1-\theta) F\left[\psi_{0}\right]+\theta F\left[\psi_{-1}\right]$ and $\psi_{-\theta} \leq \psi_{0}$ in $\mathbf{R}^{d}$, we see that, in $D_{n}$, the function $\psi_{-\theta}-M$ satisfies

$$
\begin{aligned}
& \lambda_{n}+\alpha_{n}\left(\psi_{-\theta}-M\right)+F\left[\psi_{-\theta}-M\right]-V-\alpha_{n} \psi_{0} \\
& \leq(1-\theta)\left(\lambda_{n}+F\left[\psi_{0}\right]-V\right)+\theta\left(\lambda_{n}+F\left[\psi_{-1}\right]-V\right)+\alpha_{n}\left(\psi_{-1}-\psi_{0}\right)-\alpha_{n} M<0 .
\end{aligned}
$$

Since $w_{n}$ is a solution to (4.4), one can apply the standard comparison principle in the bounded domain $D_{n}$ to conclude that $\psi_{-\theta}-M \leq w_{n}$ in $D_{n}$. Thus, we obtain the inequality $\psi_{-\theta}-M \leq w_{n}$ in $\mathbf{R}^{d}$. Letting $\theta \rightarrow 0$ and $n \rightarrow \infty$, we have $\psi_{0}-M \leq \phi^{*}$ in $\mathbf{R}^{d}$.

Now, we prove that the $A^{\phi^{*}}$-diffusion is positive recurrent. Since $\lambda^{*} \leq \lambda_{\max }$ by the definition of $\lambda_{\max }$, we observe from (ii) that

$$
\lambda^{*}+F\left[\psi_{-1}\right]-V \leq-\rho \quad \text { in } \mathbf{R}^{d} \backslash B_{R}
$$

for some $\rho, R>0$. This estimate, together with Proposition 3.4 (a), implies that $u:=\phi^{*}-\psi_{-1}$ satisfies

$$
A^{\phi^{*}} u \leq F\left[\psi_{-1}\right]-F\left[\phi^{*}\right] \leq-\rho \text { in } \mathbf{R}^{d} \backslash B_{R} .
$$

Since $u(x) \rightarrow \infty$ as $|x| \rightarrow \infty$, we conclude from Proposition 3.2 that the $A^{\phi^{*}}$-diffusion is positive recurrent.

We finally show that $\inf _{\mathbf{R}^{d}}\left(\psi_{1}-\phi^{*}\right)>-\infty$ if we assume (iii)' in addition to (i) and (ii). By choosing a smaller $\rho>0$ and a larger $R>0$ in the previous argument if necessary, one may assume that $\lambda^{*}+F\left[\psi_{1}\right]-V \geq \rho$ in $\mathbf{R}^{d} \backslash B_{R}$. Since $\lambda_{n}>\lambda^{*}-\rho$ and $\psi_{1} \geq \psi_{0}$, we see that $\psi_{1}+M$ satisfies

$$
\lambda_{n}+\alpha_{n}\left(\psi_{1}+M\right)+F\left[\psi_{1}+M\right]-V>\alpha_{n} \psi_{0} \quad \text { in } \mathbf{R}^{d} \backslash B_{R} .
$$

We claim here that $(1-\theta) w_{n}+\theta \psi_{-1} \leq \psi_{1}+M$ in $\mathbf{R}^{d}$ for all $n \geq 1$ and $\theta \in(0,1)$. It is obvious from the definition of $M$ that $(1-\theta) w_{n}+\theta \psi_{-1} \leq \psi_{1}+M$ in $\bar{B}_{R}$. Furthermore, since

$$
(1-\theta) w_{n}+\theta \psi_{-1}-\psi_{1} \leq(1-\theta)\left(-\psi_{0}(0)+\frac{2 C}{\alpha_{n}}\right)+\theta\left(\psi_{-1}-\psi_{1}\right) \rightarrow-\infty
$$

as $|x| \rightarrow \infty$, there exists an $R_{n}^{\prime}>R$ such that $(1-\theta) w_{n}+\theta \psi_{-1} \leq \psi_{1}+M$ in $\mathbf{R}^{d} \backslash B_{R_{n}^{\prime}}$. In the bounded region $D_{n}^{\prime}:=B_{R_{n}^{\prime}} \backslash \bar{B}_{R}$, one can verify that $(1-\theta) w_{n}+\theta \psi_{-1}$ satisfies

$$
\begin{aligned}
& \lambda_{n}+\alpha_{n}\left((1-\theta) w_{n}+\theta \psi_{-1}\right)+F\left[(1-\theta) w_{n}+\theta \psi_{-1}\right]-V-\alpha_{n} \psi_{0} \\
& \leq \alpha_{n} \theta\left(\psi_{-1}-\psi_{0}\right)+(1-\theta)\left(\lambda_{n}+\alpha_{n} w_{n}+F\left[w_{n}\right]-V-\alpha_{n} \psi_{0}\right)+\theta\left(\lambda_{n}+F\left[\psi_{-1}\right]-V\right) \\
& <0 \quad \text { in } \mathbf{R}^{d} \backslash B_{R} .
\end{aligned}
$$

Thus, noting this and (4.5), one can apply the standard comparison principle in bounded domain $D_{n}^{\prime}$ to conclude that $(1-\theta) w_{n}+\theta \psi_{-1} \leq \psi_{1}+M$ in $D_{n}^{\prime}$, and therefore $(1-\theta) w_{n}+\theta \psi_{-1} \leq \psi_{1}+M$ in $\mathbf{R}^{d}$. Letting $\theta \rightarrow 0$ and $n \rightarrow \infty$, we obtain $\phi^{*} \leq \psi_{1}+M$ in $\mathbf{R}^{d}$. Hence, we have completed the proof.

In order to apply the previous abstract result to our purpose, we take a triplet $\left(\psi_{-1}, \psi_{0}, \psi_{1}\right)$ satisfying (i)-(iii) of Proposition 4.3 as follows.

Proposition 4.4. Fix any $\theta \in(0,1)$ and $\gamma \in\left[1-\delta, 1+\frac{\delta}{m-1}\right]$ with $\gamma \neq 0$, and set

$$
\psi_{-1}(x):=-\frac{\varepsilon}{\gamma}\langle x\rangle^{\gamma}, \quad \psi_{0}(x):=-\frac{\theta \varepsilon}{\gamma}\langle x\rangle^{\gamma}, \quad \psi_{1}(x):=-\frac{\varepsilon^{\prime}}{1-\delta}\langle x\rangle^{1-\delta}, \quad x \in \mathbf{R}^{d},
$$

for $\varepsilon, \varepsilon^{\prime}>0$. Then the following (a) and (b) hold.

(a) There exists an $\varepsilon_{0}>0$ such that, for any $\varepsilon \in\left(0, \varepsilon_{0}\right)$,

$$
\limsup _{|x| \rightarrow \infty} \frac{F\left[\psi_{-1}\right](x)}{\langle x\rangle^{\gamma-1+\delta}}<0, \quad \limsup _{|x| \rightarrow \infty} \frac{F\left[\psi_{0}\right](x)}{\langle x\rangle^{\gamma-1+\delta}}<0 .
$$


(b) For any $\rho>0$, there exists an $\varepsilon_{1}>0$ such that $F\left[\psi_{1}\right](x) \geq-\rho$ in $\mathbf{R}^{d}$ for all $\varepsilon^{\prime} \in\left(0, \varepsilon_{1}\right)$.

Proof. We set $\psi(x):=\gamma^{-1}\langle x\rangle^{\gamma}$, and let $\varepsilon>0$. Since $D \psi(x)=\langle x\rangle^{\gamma-2} x$ and $\Delta \psi(x)=$ $d\langle x\rangle^{\gamma-2}+(\gamma-2)\langle x\rangle^{\gamma-4}|x|^{2}$ in $\mathbf{R}^{d}$, we see from (A1) and (A2) that

$$
\begin{aligned}
F[-\varepsilon \psi](x) & =\frac{\varepsilon}{2}\left\{d\langle x\rangle^{\gamma-2}+(\gamma-2)\langle x\rangle^{\gamma-4}|x|^{2}\right\}-\varepsilon(b(x) \cdot x)\langle x\rangle^{\gamma-2}+H\left(x,-\varepsilon\langle x\rangle^{\gamma-2} x\right) \\
& \leq \frac{\varepsilon}{2}(d+|\gamma-2|+K)\langle x\rangle^{\gamma-2}-\varepsilon \nu\langle x\rangle^{\gamma-1+\delta}+\frac{\varepsilon^{m}}{\nu}\langle x\rangle^{(\gamma-1) m}
\end{aligned}
$$

in $\mathbf{R}^{d}$ for some $\nu, K>0$. Noting that $\gamma-1+\delta \geq(\gamma-1) m$ if and only if $\gamma \leq 1+\frac{\delta}{m-1}$, we have

$$
F[-\varepsilon \psi](x) \leq \frac{\varepsilon}{2}(d+|\gamma-2|+K)\langle x\rangle^{\gamma-2}-\varepsilon \nu\left(1-\frac{\varepsilon^{m-1}}{\nu^{2}}\right)\langle x\rangle^{\gamma-1+\delta} .
$$

We now set $\varepsilon_{0}:=\nu^{\frac{2}{m-1}}$. Then, since $\gamma-1+\delta \geq 0$ and $\gamma-1+\delta>\gamma-2$, we conclude that, for any $\varepsilon \in\left(0, \varepsilon_{0}\right)$, there exist some $\rho, C>0$ such that

$$
F[-\varepsilon \psi](x) \leq\left\{\begin{array}{ll}
C-\rho\langle x\rangle^{\gamma-1+\delta} & \text { if } \gamma>1-\delta, \\
C\langle x\rangle^{-(1+\delta)}-\rho & \text { if } \gamma=1-\delta,
\end{array} \quad x \in \mathbf{R}^{d} .\right.
$$

The above estimate implies (a). In order to show (b), let $\rho>0$ be arbitrary. Then, in view of the first condition of (A1) and the nonnegativity of $H$, we see that

$$
\begin{aligned}
F\left[\psi_{1}\right](x) & \geq \frac{\varepsilon^{\prime}}{2}\left\{d\langle x\rangle^{-1-\delta}-(1+\delta)\langle x\rangle^{-3-\delta}|x|^{2}\right\}-\varepsilon^{\prime}(b(x) \cdot x)\langle x\rangle^{-1-\delta} \\
& \geq-\varepsilon^{\prime} C\langle x\rangle^{-1-\delta}-C \varepsilon^{\prime} \geq-2 C \varepsilon^{\prime}, \quad x \in \mathbf{R}^{d}
\end{aligned}
$$

for some $C>0$ not depending on $\varepsilon^{\prime}$. Choosing $\varepsilon_{1}>0$ so small that $2 C \varepsilon_{1}<\rho$, we obtain (c). Hence, we have completed the proof.

Using Propositions 4.3 and 4.4, we obtain the following existence theorem for (EP).

Proposition 4.5. There exists a solution $\left(\lambda^{*}, \phi^{*}\right) \in \mathbf{R} \times C^{3}\left(\mathbf{R}^{d}\right)$ of (EP) such that $\phi^{*} \in \Phi_{0}$.

Proof. Fix any $\gamma \in\left(1-\delta, 1+\frac{\delta}{m-1}\right]$, and let $\left(\psi_{-1}, \psi_{0}, \psi_{1}\right)$ be the triplet of $C^{3}$-functions of the form (4.6), where $\varepsilon, \varepsilon^{\prime}>0$ are chosen so that Proposition 4.4 holds. Then, since $\gamma-1+\delta>0$, we observe that $\left(\psi_{-1}, \psi_{0}, \psi_{1}\right)$ agrees with the conditions (i)-(iii) of Proposition 4.3. Thus, by Proposition 4.3, there exists a solution $\left(\lambda^{*}, \phi^{*}\right)$ of $(\mathrm{EP})$ such that $\inf _{\mathbf{R}^{d}}\left(\phi^{*}-\psi_{0}\right)>-\infty$. This and Proposition 4.1 yield that $\phi^{*} \in \Phi_{0}$. Hence, we have completed the proof. $\square$

Next proposition is substantial to our uniqueness result for (EP).

Proposition 4.6. Let $(\lambda, \phi) \in \mathbf{R} \times C^{3}\left(\mathbf{R}^{d}\right)$ be a solution of (EP) such that $\phi \in \Phi_{0}$, and let $X=\left(X_{t}\right)_{t \geq 0}$ be the associated $A^{\phi}$-diffusion. Then, the following (a)-(c) hold.

(a) $\sup _{n \geq 1} \mathbf{E}_{x}\left[\left|X_{T \wedge \tau_{n}}\right|^{q}\right]<\infty$ and $\mathbf{E}_{x}\left[\left|X_{T}\right|^{q}\right]<\infty$ for any $q>1, T>0$, and $x \in \mathbf{R}^{d}$, where $\tau_{n}:=\inf \left\{t>0|| X_{t} \mid>n\right\}$.

(b) $X$ is positive recurrent with an invariant probability measure $\mu$ such that

$$
\int_{\mathbf{R}^{d}}|y|^{q} \mu(d y)<\infty \text { for any } q>1 .
$$

(c) Set $\xi_{t}:=D_{p} H\left(X_{t}, D \phi\left(X_{t}\right)\right)$ for $t \geq 0$. Then $\xi \in \mathcal{A}$.

Proof. Let $\gamma=1+\frac{\delta}{m-1}$ and $\psi_{-1}, \psi_{0} \in C^{3}\left(\mathbf{R}^{d}\right)$ be as in (4.6), where $\varepsilon>0$ is taken so that Proposition 4.4 holds. Then, in view of (4.7) and the fact that $\gamma-1+\delta=m^{*} \delta$, there exist $C, \rho>0$ such that $F\left[\psi_{-1}\right] \leq C-\rho\langle x\rangle^{m^{*} \delta}$ in $\mathbf{R}^{d}$. Furthermore, since $\phi \in \Phi_{0}$, one can also see that $\phi \geq \psi_{0}-C^{\prime}$ in $\mathbf{R}^{d}$ for some $C^{\prime}>0$.

Now, we set $u:=\phi+C^{\prime}-\psi_{-1}$. Then $u(x) \geq \psi_{0}(x)-\psi_{-1}(x)=(1-\theta)(\varepsilon / \gamma)\langle x\rangle^{\gamma}$ in $\mathbf{R}^{d}$. In particular, $u(x) \rightarrow \infty$ as $|x| \rightarrow \infty$. We can also see from Propositions 3.4 (a) and 4.1 that, for any 
$q>1$,

$$
\begin{aligned}
A^{\phi} u^{q} & =q u^{q-1}\left(A^{\phi} u+\frac{q-1}{2} \frac{|D u|^{2}}{u}\right) \\
& \leq q u^{q-1}\left(F\left[\psi_{-1}\right]-F[\phi]+\frac{q-1}{2} \frac{\left|D \phi-D \psi_{-1}\right|^{2}}{u}\right) \\
& \leq q u^{q-1}\left(C-\rho\langle x\rangle^{m^{*} \delta}-\lambda+V+\frac{q-1}{2} \frac{\gamma\left(K\langle x\rangle^{\frac{\delta}{m-1}}\right)^{2}}{(1-\theta) \varepsilon\langle x\rangle^{1+\frac{\delta}{m-1}}}\right)
\end{aligned}
$$

in $\mathbf{R}^{d}$ for some $K>0$. Noting that $\frac{2 \delta}{m-1}-\left(1+\frac{\delta}{m-1}\right)<m^{*} \delta$, we have

$$
A^{\phi} u^{q} \leq q u^{q-1}\left(K^{\prime}-\frac{\rho}{2}\langle x\rangle^{m^{*} \delta}\right) \text { in } \mathbf{R}^{d}
$$

for some $K^{\prime}>0$. This implies that

$$
A^{\phi} u^{q} \leq-\rho^{\prime}\langle x\rangle^{(q-1)\left(1+\frac{\delta}{m-1}\right)+m^{*} \delta} \mathbf{1}_{\mathbf{R}^{d} \backslash B_{R}}(x)+K^{\prime \prime} \mathbf{1}_{B_{R}}(x) \text { in } \mathbf{R}^{d},
$$

for some $\rho^{\prime}, K^{\prime \prime}>0$ and $R>0$. Applying Proposition 3.2 (d) (see also Remark 3.3), we conclude that $\sup _{n \geq 1} \mathbf{E}_{x}\left[\left|X_{T \wedge \tau_{n}}\right|^{q\left(1+\frac{\delta}{m-1}\right)}\right]<\infty$ and $\mathbf{E}_{x}\left[\left|X_{T}\right|^{q\left(1+\frac{\delta}{m-1}\right)}\right]<\infty$, and that the $A^{\phi}$-diffusion is positive recurrent with an invariant probability measure $\mu$ such that $\int_{\mathbf{R}^{d}}|y|^{\alpha} \mu(y)<\infty$ with $\alpha=$ $(q-1)\left(1+\frac{\delta}{m-1}\right)$. Since $q$ can be arbitrarily large, we obtain the desired estimate.

In order to prove the last claim, we observe, in view of (2.1) and Proposition 4.1, that

$$
\left|D_{p} H(x, D \phi(x))\right|^{m^{*}} \leq C\left(|D \phi(x)|^{m-1}\right)^{m^{*}} \leq C^{\prime}\langle x\rangle^{m^{*} \delta}, \quad x \in \mathbf{R}^{d}
$$

for some $C, C^{\prime}>0$. In particular,

$$
\mathbf{E}_{x}\left[\int_{0}^{T}\left|\xi_{t}\right|^{m^{*}} d t\right] \leq \mathbf{E}_{x}\left[\int_{0}^{T} C^{\prime}\left\langle X_{t}\right\rangle^{m^{*} \delta} d t\right] \leq C^{\prime} \sup _{0 \leq t \leq T} \mathbf{E}_{x}\left[\left\langle X_{t}\right\rangle^{m^{*} \delta}\right]<\infty
$$

for all $T>0$ and $x \in \mathbf{R}^{d}$, which implies that $\xi \in \mathcal{A}$. Hence, we have completed the proof. $\square$

REMARK 4.7. If $m \geq 2$ in (A2), we are able to obtain a better integrability of $\mu$ in Proposition 4.6. More precisely, one has $\int_{\mathbf{R}^{d}} \exp \left(\theta|y|^{1+\frac{\delta}{m-1}}\right) \mu(d y)<\infty$ for some $\theta>0$. To prove this, we apply Proposition 3.4 (b) to see that

$$
A^{\phi} e^{\kappa\left(\phi-\psi_{-1}\right)} \leq \kappa e^{\kappa\left(\phi-\psi_{-1}\right)}\left(F\left[\psi_{-1}\right]-F[\phi]+1\right) \leq-\rho \kappa e^{\kappa\left(\phi-\psi_{-1}\right)} \quad \text { in } \quad \mathbf{R}^{d} \backslash B_{R}
$$

for some $\rho, R>0$. Since $e^{\kappa\left(\phi-\psi_{-1}\right)} \geq e^{\kappa(1-\theta) \varepsilon\langle x\rangle^{\gamma}}$ with $\gamma=1+\frac{\delta}{m-1}$, we obtain the desired integrability of $\mu$ in view of Proposition 3.2 (d).

We now prove the uniqueness of eigenfunctions to (EP) with $\lambda=\lambda_{\max }$.

Proposition 4.8. Let $(\lambda, \phi)$ be a solution of $(E P)$ such that $\phi \in \Phi_{0}$. Then, $\lambda=\lambda_{\max }$. Moreover, if $\phi_{\max } \in C^{2}\left(\mathbf{R}^{d}\right)$ is any eigenfunction of (EP) associated with $\lambda_{\max }$, then $\phi-\phi_{\max }$ is constant in $\mathbf{R}^{d}$.

Proof. We first show that $\lambda=\lambda_{\max }$. Let $X=\left(X_{t}\right)_{t \geq 0}$ be the $A^{\phi}$-diffusion and set $\xi_{t}:=$ $D_{p} H\left(X_{t}, D \phi\left(X_{t}\right)\right)$. Then, in view of Proposition 4.6, we see that $\xi \in \mathcal{A}$. Furthermore, from Lemma B.2 in Appendix B, we have

$$
\begin{aligned}
\lambda_{\max } T+\phi_{\max }(x)-\mathbf{E}_{x}\left[\phi_{\max }\left(X_{T}\right)\right] & \leq \mathbf{E}_{x}\left[\int_{0}^{T}\left\{L\left(X_{t}, \xi_{t}\right)+V\left(X_{t}\right)\right\} d t\right], \\
& =\lambda T+\phi(x)-\mathbf{E}_{x}\left[\phi\left(X_{T}\right)\right]
\end{aligned}
$$

for any $T>0$ and $x \in \mathbf{R}^{d}$. In particular, we obtain

$$
\left(\lambda_{\max }-\lambda\right) T+\left(\phi_{\max }-\phi\right)(x) \leq \mathbf{E}_{x}\left[\left(\phi_{\max }-\phi\right)\left(X_{T}\right)\right], \quad T>0, \quad x \in \mathbf{R}^{d} .
$$


Since both $\phi_{\max }$ and $\phi$ are of polynomial growth, we see by virtue of Propositions 3.1 and 4.6 that

$$
\mathbf{E}_{x}\left[\left(\phi_{\max }-\phi\right)\left(X_{T}\right)\right] \longrightarrow \int_{\mathbf{R}^{d}}\left(\phi_{\max }-\phi\right)(y) \mu(d y)<\infty \quad \text { as } T \rightarrow \infty
$$

Thus, dividing both sides of (4.8) by $T$ and letting $T \rightarrow \infty$, we obtain $\lambda_{\max } \leq \lambda$. Since $\lambda_{\max } \geq \lambda$ is obvious from the definition of $\lambda_{\max }$, we conclude that $\lambda=\lambda_{\max }$.

We next show that $\phi_{\max }-\phi$ is constant in $\mathbf{R}^{d}$. Since $\lambda=\lambda_{\max }$, we see from (4.8) that

$$
\left(\phi_{\max }-\phi\right)(x) \leq \mathbf{E}_{x}\left[\left(\phi_{\max }-\phi\right)\left(X_{T}\right)\right], \quad x \in \mathbf{R}^{d}, \quad T>0 .
$$

Sending $T \rightarrow \infty$ and then taking the supremum over all $x \in \mathbf{R}^{d}$, we have

$$
\sup _{\mathbf{R}^{d}}\left(\phi_{\max }-\phi\right) \leq \int_{\mathbf{R}^{d}}\left(\phi_{\max }-\phi\right)(y) \mu(d y)<\infty, \quad x \in \mathbf{R}^{d}
$$

From this estimate, together with the fact that $\operatorname{supp} \mu=\mathbf{R}^{d}$ (see, e.g, [30, Theorem 4.8.4]), we conclude that $\phi_{\max }-\phi$ is constant in $\mathbf{R}^{d}$. Hence, we have completed the proof.

We finally show that $\phi^{*} \in \Phi_{1}$ if we replace (A3a) by (A3b).

Proposition 4.9. Assume (A1) with $\delta>0$, (A2), and (A3b), and let $\left(\lambda^{*}, \phi^{*}\right) \in \mathbf{R} \times C^{3}\left(\mathbf{R}^{d}\right)$ be the solution of (EP) in Proposition 4.5. Then $\phi^{*} \in \Phi_{1}$.

Proof. We choose the triplet of $C^{3}$-functions $\left(\psi_{-1}, \psi_{0}, \psi_{1}\right)$ as in the proof of Proposition 4.5. It suffices to verify that $\psi_{1}$ satisfies (iii)' of Proposition 4.3. Since $V(x) \rightarrow 0$ as $|x| \rightarrow \infty$, we see from Proposition 4.4 (b) that the validity of (iii) ${ }^{\prime}$ is reduced to verifying that $\lambda^{*}>0$. Let $X=\left(X_{t}\right)_{t \geq 0}$ be the $A^{\phi^{*}}$-diffusion and set $\xi_{t}:=D_{p} H\left(X_{t}, D \phi^{*}\left(X_{t}\right)\right)$. Since $\xi \in \mathcal{A}$ in view of Proposition 4.6, we see from Lemma B.2 in Appendix B that, for any $T>0$ and $x \in \mathbf{R}^{d}$,

$$
\lambda^{*} T+\phi^{*}(x)=\mathbf{E}_{x}\left[\int_{0}^{T}\left\{L\left(X_{t}, \xi_{t}\right)+V\left(X_{t}\right)\right\} d t+\phi^{*}\left(X_{T}\right)\right] .
$$

Dividing both sides by $T$ and then letting $T \rightarrow \infty$, we have

$$
\lambda^{*} \geq \lim _{T \rightarrow \infty} \frac{1}{T} \mathbf{E}_{x}\left[\int_{0}^{T} V\left(X_{t}\right) d t+\phi^{*}\left(X_{T}\right)\right]=\int_{\mathbf{R}^{d}} V(y) \mu(d y)>0,
$$

where we have used the fact that $\mathbf{E}_{x}\left[\phi^{*}\left(X_{T}\right)\right]$ converges to a constant as $T \rightarrow \infty$. Hence, we have completed the proof.

The proof of Theorem 2.1 is now easy. Indeed, claims (a) and (b) are direct consequences of Propositions 4.5 and 4.8. Claim (c) is nothing but Proposition 4.9.

5. Proof of Theorem 2.2. The goal of this section is to prove Theorem 2.2. In what follows, we assume (A1) with $\delta>0$, (A2), and (A3b), together with $g \in \Psi_{1+\frac{\delta}{m-1}}$. Note that the value function $u(T, x)$ defined by (2.8) belongs to $C^{1,2}\left((0, \infty) \times \mathbf{R}^{d}\right) \cap C\left([0, \infty) \times \mathbf{R}^{d}\right)$ and is a solution to the Cauchy problem

$$
\begin{cases}u_{t}+F[u]-V=0 & \text { in }(0, \infty) \times \mathbf{R}^{d} \\ u(0, \cdot)=g & \text { in } \mathbf{R}^{d}\end{cases}
$$

where $u_{t}:=\partial u / \partial t$. We prove this fact in Appendix C.

Let $\left(\lambda^{*}, \phi^{*}\right)$ be the solution of (EP) in Theorem 2.1, and set

$$
w(T, x):=u(T, x)-\lambda^{*} T-\phi^{*}(x), \quad T \geq 0, \quad x \in \mathbf{R}^{d} .
$$

Then, convergence (2.9) is reduced to showing that the family $\{w(T, \cdot)\}_{T>0}$ converges to a constant $c \in \mathbf{R}$ in $C\left(\mathbf{R}^{d}\right)$ as $T \rightarrow \infty$. 
Proposition 5.1. Suppose that either (i) or (ii) in Theorem 2.2 holds. Then, the family $\{w(T, \cdot)\}_{T>0}$ is uniformly bounded on any compact subset of $\mathbf{R}^{d}$.

Proof. We first verify that $\{w(T, \cdot)\}_{T>0}$ is bounded above uniformly on any compact subset of $\mathbf{R}^{d}$. Let $X=\left(X_{t}\right)_{t \geq 0}$ be the $A^{\phi^{*}}$-diffusion and set $\xi_{t}^{*}:=D_{p} H\left(X_{t}, D \phi^{*}\left(X_{t}\right)\right)$. Then, since $\xi^{*} \in \mathcal{A}$, we see from the definition of $u(T, x)$ and Lemma B.2 in Appendix B that

$$
\begin{aligned}
u(T, x) & \leq \mathbf{E}_{x}\left[\int_{0}^{T}\left(L\left(X_{t}, \xi_{t}^{*}\right)+V\left(X_{t}\right)\right) d t+g\left(X_{T}\right)\right] \\
& =\lambda^{*} T+\phi^{*}(x)+\mathbf{E}_{x}\left[\left(g-\phi^{*}\right)\left(X_{T}\right)\right]
\end{aligned}
$$

for all $T>0$ and $x \in \mathbf{R}^{d}$. This implies that

$$
w(T, x)=u(T, x)-\lambda^{*} T-\phi^{*}(x) \leq \mathbf{E}_{x}\left[\left(g-\phi^{*}\right)\left(X_{T}\right)\right] .
$$

Since $\mathbf{E}_{x}\left[\left(g-\phi^{*}\right)\left(X_{T}\right)\right]$ converges to $\int_{\mathbf{R}^{d}}\left(g-\phi^{*}\right)(y) \mu(d y)<\infty$ as $T \rightarrow \infty$ uniformly on any compact subset of $\mathbf{R}^{d}$, we conclude that $\{w(T, \cdot)\}_{T>0}$ is bounded above uniformly on any compact subset of $\mathbf{R}^{d}$.

In order to see that $\{w(T, \cdot)\}_{T>0}$ is bounded below uniformly on any compact subset of $\mathbf{R}^{d}$, we first assume (i) in Theorem 2.2, namely, $g \in \Psi_{1-\delta}$. Then, since $\phi^{*} \in \Phi_{1}$ by virtue of Theorem 2.1 , we see that $g \geq \phi^{*}-C$ in $\mathbf{R}^{d}$ for some $C>0$. This and Lemma B.2 imply that

$$
u(T, x) \geq \inf _{\xi \in \mathcal{A}} \mathbf{E}_{x}\left[\int_{0}^{T}\left(L\left(X_{t}^{\xi}, \xi_{t}\right)+V\left(X_{t}^{\xi}\right)\right) d t+\phi^{*}\left(X_{T}^{\xi}\right)\right]-C=\lambda^{*} T+\phi^{*}(x)-C
$$

for all $T>0$ and $x \in \mathbf{R}^{d}$. Hence, $w(T, \cdot) \geq-C$ in $\mathbf{R}^{d}$ for any $T>0$, and the claim is valid.

Suppose next that (ii) holds in Theorem 2.2, namely, $g \in \Psi_{m^{*}}$ and $\delta=1$ in (A1). Since $\phi^{*} \in \Phi_{1}$, we may assume, by adding a constant to $\phi^{*}$ if necessary, that $\phi^{*} \leq 0$ in $\mathbf{R}^{d}$. Furthermore, by assumption (ii), for any $\varepsilon>0$, there exists a $C_{\varepsilon}>0$ such that $g(x) \geq-\varepsilon\langle x\rangle^{m^{*}}-C_{\varepsilon}$ in $\mathbf{R}^{d}$. We set $\psi:=-\varepsilon\langle x\rangle^{m^{*}}-C_{\varepsilon}$. Then, in view of (4.7) with $\gamma=1+\frac{\delta}{m-1}=m^{*}$, one can choose an $\varepsilon$ such that $F[\psi] \leq C-\rho\langle x\rangle^{m^{*}}$ in $\mathbf{R}^{d}$ for some $\rho, C>0$. Hereafter, we fix such $\varepsilon>0$.

Now, for given $k, K>0$, we define the function $v:[0, \infty) \times \mathbf{R}^{d} \rightarrow \mathbf{R}$ by

$$
v(t, x):=\left(1-e^{-k t}\right)\left(\phi^{*}(x)-K\right)+e^{-k t} \psi(x)+\lambda^{*} t, \quad(t, x) \in[0, \infty) \times \mathbf{R}^{d} .
$$

We claim that $v$ is a subsolution of the Cauchy problem (5.1) provided $k$ is sufficiently small and $K$ is sufficiently large. Indeed, in view of the convexity of $\phi \mapsto F[\phi]$ and the fact that $\phi^{*} \leq 0$ and $V \geq 0$ in $\mathbf{R}^{d}$, we observe that

$$
\begin{aligned}
v_{t}+F[v]-V & \leq k e^{-k t}\left(\phi^{*}-K\right)-k e^{-k t} \psi+\lambda^{*}+\left(1-e^{-k t}\right) F\left[\phi^{*}\right]+e^{-k t} F[\psi]-V \\
& =e^{-k t}\left(k \phi^{*}-k K-k \psi+F[\psi]\right)+\left(1-e^{-k t}\right)\left(V-\lambda^{*}\right)+\lambda^{*}-V \\
& \leq e^{-k t}\left\{\left(k C_{\varepsilon}-k K+C+\lambda^{*}\right)+(k \varepsilon-\rho)\langle x\rangle^{m^{*}}\right\} .
\end{aligned}
$$

Choosing $k>0$ so small that $k \varepsilon<\rho$ and $K>0$ so large that $k C_{\varepsilon}-k K+C+\lambda^{*}<0$, we obtain the subsolution property of $v$.

Since $\sup _{0 \leq t \leq T, x \in \mathbf{R}^{d}}|v(t, x)|\langle x\rangle^{-\left(1+\frac{\delta}{m-1}\right)}<\infty$ for all $T>0$, one can apply Theorem C.1 (a) in Appendix $\mathrm{C}$ to conclude that $v \leq u$ in $[0, \infty) \times \mathbf{R}^{d}$. Thus, we have

$$
w(T, x) \geq v(T, x)-\lambda^{*} T-\phi^{*}(x) \geq e^{-k T}\left(\psi-\phi^{*}\right)(x)-K
$$

for any $T>0$ and $x \in \mathbf{R}^{d}$. This yields that $\{w(T, \cdot)\}_{T>0}$ is bounded below on any compact subset of $\mathbf{R}^{d}$. Hence, we have completed the proof. 
Proposition 5.2. Suppose that either (i) or (ii) in Theorem 2.2 holds. Then, there exists a constant $c \in \mathbf{R}$ such that, for any diverging sequence $\left\{S_{j}\right\}$, one has $w\left(S_{j}, x\right) \rightarrow c$ in $C\left(\mathbf{R}^{d}\right)$ as $j \rightarrow \infty$.

Proof. Since $\{w(T, \cdot)\}_{T>0}$ is equi-continuous on any compact subset of $\mathbf{R}^{d}$ by Theorem C.1 (c) in Appendix C, we observe, together with Proposition 5.1, that there exists a diverging sequence $\left\{S_{j}\right\}$ such that $\left\{w\left(S_{j}, \cdot\right)\right\}_{j \geq 1}$ converges to a function in $C\left(\mathbf{R}^{d}\right)$. Hence, in order to verify the claim, it suffices to prove that any converging sequence $\left\{w\left(S_{j}, \cdot\right)\right\}_{j \geq 1}$ in $C\left(\mathbf{R}^{d}\right)$ has the same limit $c \in \mathbf{R}$. We first note that $w$ satisfies

$$
w_{t}-A^{\phi^{*}} w+\tilde{H}(x, D w)=0 \quad \text { in }(0, \infty) \times \mathbf{R}^{d}, \quad w(0, \cdot)=g-\phi^{*} \quad \text { in } \mathbf{R}^{d},
$$

where $w_{t}:=\partial w / \partial t$, and $\tilde{H}(x, p)$ is defined by

$$
\tilde{H}(x, p):=H\left(x, D \phi^{*}(x)+p\right)-H\left(x, D \phi^{*}(x)\right)-D_{p} H\left(x, D \phi^{*}(x)\right) \cdot p, \quad x, p \in \mathbf{R}^{d} .
$$

Note that $\tilde{H} \geq 0$ in $\mathbf{R}^{d} \times \mathbf{R}^{d}$, which comes from the convexity of $H(x, p)$ with respect to $p$. Let $X=\left(X_{t}\right)_{t \geq 0}$ be the $A^{\phi^{*}}$-diffusion and apply Ito's formula to $w\left(T+S-t, X_{t}\right)$. Then,

$$
\begin{aligned}
& \mathbf{E}_{x}\left[w\left(T+S-T \wedge \tau_{n}, X_{T \wedge \tau_{n}}\right)\right]-w(T+S, x) \\
& \quad=\mathbf{E}_{x}\left[\int_{0}^{T \wedge \tau_{n}}\left(-w_{t}\left(T+S-t, X_{t}\right)+A^{\phi^{*}} w\left(T+S-t, X_{t}\right)\right) d t\right] \geq 0,
\end{aligned}
$$

where $\tau_{n}:=\inf \left\{t>0|| X_{t} \mid>n\right\}$. Since $\sup _{t \in[0, S+T]}|w(t, x)| \leq C\langle x\rangle^{q}$ in $\mathbf{R}^{d}$ for some $C, q>0$ and $\sup \mathbf{E}_{x}\left[\left|X_{T \wedge \tau_{n}}\right|^{q}\right]<\infty$ by virtue of Proposition 4.6 , we observe by sending $n \rightarrow \infty$ in the above $n \geq 1$. estimate that

$$
w(T+S, x) \leq \mathbf{E}_{x}\left[w\left(S, X_{T}\right)\right], \quad T, S>0, \quad x \in \mathbf{R}^{d}
$$

Now, let $\left\{S_{j}\right\}$ be any diverging sequence such that $\left\{w\left(S_{j}, \cdot\right)\right\}$ converges in $C\left(\mathbf{R}^{d}\right)$ to a function $w_{\infty} \in C\left(\mathbf{R}^{d}\right)$ as $j \rightarrow \infty$. Fix any $S>0, x \in \mathbf{R}^{d}$, and set $T_{j}:=S_{j}-S$. Then, in view of (5.2), we see that $w\left(S_{j}, x\right)=w\left(T_{j}+S, x\right) \leq \mathbf{E}_{x}\left[w\left(S, X_{T_{j}}\right)\right]$ for any $j \geq 1$. Letting $j \rightarrow \infty$, we have

$$
w_{\infty}(x)=\lim _{j \rightarrow \infty} w\left(S_{j}, x\right) \leq \lim _{j \rightarrow \infty} \mathbf{E}_{x}\left[w\left(S, X_{T_{j}}\right)\right]=\int_{\mathbf{R}^{d}} w(S, y) \mu(d y) .
$$

Put $S=S_{j}$ in the above inequality and consider the limit as $j \rightarrow \infty$. Then, since $w\left(S_{j}, \cdot\right)$ converges to $w_{\infty}$ locally uniformly in $\mathbf{R}^{d}$ and $|w(T, x)| \leq h(x)$ in $[1, \infty) \times \mathbf{R}^{d}$ for some $h \in C_{\text {pol }}\left(\mathbf{R}^{d}\right)$, we see by the dominated convergence theorem that

$$
w_{\infty}(x) \leq \lim _{j \rightarrow \infty} \int_{\mathbf{R}^{d}} w\left(S_{j}, y\right) \mu(d y)=\int_{\mathbf{R}^{d}} w_{\infty}(y) \mu(d y)<\infty .
$$

This yields that $w_{\infty}(x)=\sup _{\mathbf{R}^{d}} w_{\infty}<\infty$ in $\mathbf{R}^{d}$. Hence, $w_{\infty}$ is constant in $\mathbf{R}^{d}$.

We next show that the limit of $\left\{w\left(S_{j}, \cdot\right)\right\}$ does not depend on the choice of $\left\{S_{j}\right\}$. Let $\left\{S_{j}\right\}$ and $\left\{T_{j}\right\}$ be any diverging sequences such that $\left\{w\left(S_{j}, \cdot\right)\right\}$ and $\left\{w\left(T_{j}, \cdot\right)\right\}$ converge in $C\left(\mathbf{R}^{d}\right)$ to some constants $c_{1}$ and $c_{2}$, respectively. Then, putting $T=T_{j}-S$ in (5.2) and sending $j \rightarrow \infty$, we have

$$
c_{2}=\lim _{j \rightarrow \infty} w\left(T_{j}, y\right) \leq \lim _{j \rightarrow \infty} \mathbf{E}_{x}\left[w\left(S, X_{T_{j}}\right)\right]=\int_{\mathbf{R}^{d}} w(S, y) \mu(d y) .
$$

We then put $S=S_{j}$ and let $j \rightarrow \infty$ to obtain

$$
c_{2} \leq \lim _{j \rightarrow \infty} \int_{\mathbf{R}^{d}} w\left(S_{j}, y\right) \mu(d y)=c_{1} .
$$


Thus, $c_{2} \leq c_{1}$. Changing the role of $\left\{S_{j}\right\}$ and $\left\{T_{j}\right\}$, we also have $c_{1} \leq c_{2}$. Hence, $c_{1}=c_{2}$, and we have completed the proof.

We close this section with the proof of Theorem 2.2.

Proof of Theorem 2.2. The latter claim, i.e. convergence (2.9) follows from Proposition 5.2. It remains to verify that $\Lambda(x)=\lambda_{\max }$ for all $x \in \mathbf{R}^{d}$. Let $u(T, x)$ be the value function in (2.8). Then, we have

$$
\lambda^{*}=\lim _{T \rightarrow \infty} \frac{u(T, x)}{T} \leq \Lambda(x) \leq \lambda^{*}, \quad x \in \mathbf{R}^{d} .
$$

Indeed, the first equality holds in view of Proposition 5.2. The inequality in the middle follows from the very definitions of $\Lambda(x)$ and $u(T, x)$. To see the last inequality, let $X=\left(X_{t}\right)_{t \geq 0}$ be the $A^{\phi^{*}}$-diffusion and remind Lemma B.2 to see that

$$
\lambda^{*} T+\phi^{*}(x)=\mathbf{E}_{x}\left[\int_{0}^{T}\left(L\left(X_{t}, \xi_{t}^{*}\right)+V\left(X_{t}\right)\right) d t+\phi^{*}\left(X_{T}\right)\right]
$$

for any $T>0$ and $x \in \mathbf{R}^{d}$, where $\xi_{t}^{*}:=D_{p} H\left(X_{t}, D \phi^{*}\left(X_{t}\right)\right)$. Dividing both sides by $T$ and then sending $T \rightarrow \infty$, we have

$$
\lambda^{*}=\limsup _{T \rightarrow \infty} \frac{1}{T} \mathbf{E}_{x}\left[\int_{0}^{T}\left(L\left(X_{t}, \xi_{t}\right)+V\left(X_{t}\right)\right) d t+g\left(X_{T}\right)+\left(\phi^{*}-g\right)\left(X_{T}\right)\right] \geq \Lambda(x)
$$

for all $x \in \mathbf{R}^{d}$, where we have used the fact that $\mathbf{E}_{x}\left[\left(\phi^{*}-g\right)\left(X_{T}\right)\right]$ converges to a constant as $T \rightarrow \infty$. Hence, we obtain $\lambda^{*}=\Lambda(x)$.

6. Proof of Theorem 2.3. In this section we prove Theorem 2.3. In what follows, we assume (A1), (A2), and (A3c). We denote by $\lambda_{\max }(\beta)$ the generalized principal eigenvalue of the ergodic problem $\left(\mathrm{EP}_{\beta}\right)$ given in Section 2. Note that $\lambda_{\max }(0)=0$. Indeed, suppose first that $\delta>0$. Then, since $(\lambda, \phi):=(0,0)$ is a solution of $\left(\mathrm{EP}_{\beta}\right)$ with $\beta=0$ such that $\phi \in \Phi_{0}$, and therefore $\lambda_{\max }=0$ by Theorem 2.1. Suppose next that $\delta=0$. Then, by virtue of [20, Proposition 5.11], we obtain $\lambda_{\max }(0)=0$.

We first remark the monotonicity and concavity of $\lambda_{\max }(\beta)$ with respect to $\beta$ that can be deduced from the nonnegativity of $V$ and the convexity of $H(x, p)$ in $p$.

Proposition 6.1. Let $0 \leq \beta_{0} \leq \beta_{1}$. Then $\lambda_{\max }\left(\beta_{0}\right) \leq \lambda_{\max }\left(\beta_{1}\right)$ and $(1-\theta) \lambda_{\max }\left(\beta_{0}\right)+$ $\theta \lambda_{\max }\left(\beta_{1}\right) \leq \lambda_{\max }\left((1-\theta) \beta_{0}+\theta \beta_{1}\right)$ for all $\theta \in[0,1]$.

Proof. Since any subsolution $(\lambda, \phi) \in \mathbf{R} \times C^{3}\left(\mathbf{R}^{d}\right)$ of $\left(\mathrm{EP}_{\beta}\right)$ with $\beta=\beta_{0}$ is a subsolution of $\left(\mathrm{EP}_{\beta}\right)$ with $\beta=\beta_{1}$, one has $\lambda \leq \lambda_{\max }\left(\beta_{1}\right)$. Taking the supremum over all $\lambda$ such that $(\lambda, \phi)$ is a subsolution of $\left(\mathrm{EP}_{\beta}\right)$ with $\beta=\beta_{0}$, we obtain $\lambda_{\max }\left(\beta_{0}\right) \leq \lambda_{\max }\left(\beta_{1}\right)$.

Next, let $\phi_{0}, \phi_{1} \in C^{3}\left(\mathbf{R}^{d}\right)$ be any subsolutions of $\left(\mathrm{EP}_{\beta}\right)$ with $\beta=\beta_{0}$ and $\beta=\beta_{1}$, respectively. We set $\phi_{\theta}:=(1-\theta) \phi_{0}+\theta \phi_{1}$ and $\lambda_{\theta}:=(1-\theta) \lambda_{\max }\left(\beta_{0}\right)+\theta \lambda_{\max }\left(\beta_{1}\right)$. Then, by the convexity of $H(x, p)$ in $p$, one can verify that $\left(\lambda_{\theta}, \phi_{\theta}\right)$ is a subsolution of $\left(\mathrm{EP}_{\beta}\right)$ with $\beta=(1-\theta) \beta_{0}+\theta \beta_{1}$. This implies the concavity of $\lambda_{\max }(\beta)$ with respect to $\beta$. Hence, we have completed the proof.

We next derive the upper bound of $\lambda_{\max }(\beta)$.

Proposition 6.2. There exists a $C>0$ such that $\lambda_{\max }(\beta) \leq C\left(1+\beta^{\frac{m \delta}{m \delta+\eta(m-1)}}\right)$ for all $\beta \geq 0$.

Proof. We may assume without loss of generality that $\beta>1$. Let $(\lambda, \phi) \in \mathbf{R} \times C^{3}\left(\mathbf{R}^{d}\right)$ be any subsolution of $\left(\mathrm{EP}_{\beta}\right)$, and fix any test function $\zeta \in C^{\infty}\left(\mathbf{R}^{d}\right)$ such that $\zeta \geq 0$ in $\mathbf{R}^{d}$, supp $\zeta \subset B_{1}$, and $\int_{\mathbf{R}^{d}} \zeta(x)^{m^{*}} d x=1$, where $m^{*}:=m /(m-1)$. Then, we have

$$
\lambda+\frac{1}{2} \int_{\mathbf{R}^{d}} D\left(\zeta^{m^{*}}\right) \cdot D \phi d x+\int_{\mathbf{R}^{d}} \zeta^{m^{*}} b \cdot D \phi d x+\int_{\mathbf{R}^{d}} \zeta^{m^{*}} H(x, D \phi) d x \leq \beta \int_{\mathbf{R}^{d}} \zeta^{m^{*}} V d x,
$$

where we drop the variable $x$ in the integrands. From (A2), there exists a $\nu>0$ such that

$$
\int_{\mathbf{R}^{d}} \zeta^{m^{*}} H(x, D \phi) d x \geq \nu \int_{\mathbf{R}^{d}} \zeta^{m^{*}}|D \phi|^{m} d x
$$


Furthermore, we observe by Young's inequality that

$$
\begin{aligned}
\frac{1}{2} \int_{\mathbf{R}^{d}} D\left(\zeta^{m^{*}}\right) \cdot D \phi d x & =\frac{m^{*}}{2} \int_{\mathbf{R}^{d}} \zeta^{m^{*}-1} D \zeta \cdot D \phi d x=\frac{m^{*}}{2} \int_{\mathbf{R}^{d}}\left(\zeta^{\frac{1}{m-1}} D \phi\right) \cdot D \zeta d x \\
& \geq-\frac{\nu}{2} \int_{\mathbf{R}^{d}} \zeta^{m^{*}}|D \phi|^{m} d x-C \int_{\mathbf{R}^{d}}|D \zeta|^{m^{*}} d x
\end{aligned}
$$

for some $C>0$ depending only on $m$ and $\nu$. In what follows, $C$ denotes various constants depending only on $m$ and $\nu$. Similarly as above, we also have

$$
\int_{\mathbf{R}^{d}} \zeta^{m^{*}} b \cdot D \phi d x \geq-\frac{\nu}{2} \int_{\mathbf{R}^{d}} \zeta^{m^{*}}|D \phi|^{m} d x-C \int_{\mathbf{R}^{d}} \zeta^{m^{*}}|b|^{m^{*}} d x .
$$

Gathering these estimates, we obtain

$$
\lambda \leq C \int_{\mathbf{R}^{d}}|D \zeta|^{m^{*}} d x+C \int_{\mathbf{R}^{d}} \zeta^{m^{*}}|b|^{m^{*}} d x+\beta \int_{\mathbf{R}^{d}} \zeta^{m^{*}} V d x .
$$

Now, we set $\zeta_{y}(x):=\zeta(x-y)$ for $x, y \in \mathbf{R}^{d}$. Then, replacing the above $\zeta$ by $\zeta_{y}$ and using $(\mathrm{A} 1)$ and (A3), we have

$$
\begin{aligned}
\lambda & \leq C\left(1+\int_{B_{1}} \zeta(x)^{m^{*}}|b(x+y)|^{m^{*}} d x+\beta \int_{B_{1}} \zeta(x)^{m^{*}} V(x+y) d x\right) \\
& \leq C\left(1+\langle y\rangle^{m^{*} \delta}+\beta\langle y\rangle^{-\eta}\right)
\end{aligned}
$$

for any $y \in \mathbf{R}^{d}$. We fix any $\theta>0$ and choose a $y \in \mathbf{R}^{d}$ such that $\langle y\rangle=\beta^{\theta}$. Then, from the above estimate, we have $\lambda \leq C\left(1+\beta^{m^{*} \delta \theta}+\beta^{1-\eta \theta}\right)$. Choosing $\theta$ so that $m^{*} \delta \theta=1-\eta \theta$, namely, $\theta=\left(m^{*} \delta+\eta\right)^{-1}$, we have $\lambda \leq C\left(1+\beta^{\frac{m^{*} \delta}{m^{*} \delta+\eta}}\right)=C\left(1+\beta^{\frac{m \delta}{m \delta+\eta(m-1)}}\right)$. Since $\lambda<\lambda_{\max }(\beta)$ is arbitrary, we obtain the desired estimate.

Now, we study the lower bound of $\lambda_{\max }(\beta)$. The proof is divided whether $\delta>0$ or $\delta=0$. We first consider the case where $\delta>0$.

Proposition 6.3. Suppose that $\delta>0$ in (A1). Then there exist $\nu, C>0$ such that $\lambda_{\max }(\beta) \geq$ $\nu \beta^{\frac{m \delta}{m \delta+\eta(m-1)}}-C$ for all $\beta \geq 0$.

Proof. We set $\psi:=\gamma\langle x\rangle^{\gamma}$ with $\gamma=1+\frac{\delta}{m-1}$. Then, in view of (4.7) and recalling that $\gamma-1+\delta=m^{*} \delta$, we have $F[-\varepsilon \psi](x) \leq C-\rho\langle x\rangle^{m^{*} \delta}$ in $\mathbf{R}^{d}$ for some $\varepsilon, C, \rho>0$. Since $V(x) \geq c\langle x\rangle^{-\eta}$ in $\mathbf{R}^{d}$ for some $c>0$, we obtain

$$
F[-\varepsilon \psi]-\beta V \leq C-\rho\langle x\rangle^{m^{*} \delta}-\beta c\langle x\rangle^{-\eta} \text { in } \mathbf{R}^{d}
$$

We now set $\langle r\rangle:=\left(1+r^{2}\right)^{1 / 2}$ and $f(r):=\rho\langle r\rangle^{m^{*} \delta}+\beta c\langle r\rangle^{-\eta}$ for $r \geq 0$. Then, by direct computations, we observe that $f^{\prime}(r)=\langle r\rangle^{-\eta-2} r\left(\rho m^{*} \delta\langle r\rangle^{m^{*} \delta+\eta}-\beta c \eta\right)$ for $r \geq 0$. This implies that, if $\beta>\rho m^{*} \delta /(c \eta)=: c_{1}$, then $f(r)$ attains its minimum at $r^{*}>0$ such that $\left\langle r^{*}\right\rangle=\left(\beta / c_{1}\right)^{\frac{1}{m^{*} \delta+\eta}}$, and $f\left(r^{*}\right)=\nu \beta^{\frac{m^{*} \delta}{m^{*} \delta+\eta}}=\nu \beta^{\frac{m \delta}{m \delta+\eta(m-1)}}$ for some constant $\nu>0$ not depending on $\beta$. Thus, we obtain

$$
F[-\varepsilon \psi]-\beta V \leq C-f(|x|) \leq C-f\left(r^{*}\right)=C-\nu \beta^{\frac{m \delta}{m \delta+\eta(m-1)}} \quad \text { in } \quad \mathbf{R}^{d}
$$

for any $\beta>c_{1}$. In particular, $(\lambda, \phi)=\left(\nu \beta^{\frac{m \delta}{m \delta+\eta(m-1)}}-C, \varepsilon \psi\right)$ is a subsolution of $\left(\mathrm{EP}_{\beta}\right)$. Thus, by the definition of $\lambda_{\max }(\beta)$, we conclude that $\lambda_{\max }(\beta) \geq \nu \beta^{\frac{m \delta}{m \delta+\eta(m-1)}}-C$. This inequality is valid for any $\beta \geq 0$ if we replace $C$ by a larger one. Hence, we have completed the proof.

REMARK 6.4. Similarly as in the proof of [20, Proposition 5.4], one can show that $\lambda_{\max }(\beta)$ is differentiable with respect to $\beta$ for any $\beta>0$ and

$$
\frac{d}{d \beta} \lambda_{\max }(\beta)=\int_{\mathbf{R}^{d}} V(y) \mu_{\beta}(d y)>0,
$$

where $\mu_{\beta}$ stands for the invariant probability measure for the associated $A^{\phi^{*}}$-diffusion. 
We next prove the positivity of $\lambda_{\max }(\beta)$ in the case where $\delta=0$.

Proposition 6.5. Suppose that $\delta=0$ in (A1). Then, $\lambda_{\max }(\beta)>0$ for all $\beta>0$.

Proof. Since $\lambda_{\max }(0)=0$ and $\lambda_{\max }(\beta)$ is concave in $\beta$, it suffices to verify that $\lambda_{\max }(\beta)>0$ for some $\beta>0$. In order to prove this, we set $\psi:=\langle x\rangle$. Then, similarly as in the proof of Proposition 4.4, we see that $F[-\varepsilon \psi](x) \leq C\langle x\rangle^{-1}-\rho$ in $\mathbf{R}^{d}$ for some $\varepsilon, C, \rho>0$, which implies that

$$
F[-\varepsilon \psi]-\beta V \leq-\rho-\left(\beta c\langle x\rangle^{-\eta}-C\langle x\rangle^{-1}\right) \text { in } \mathbf{R}^{d}
$$

for some $c>0$. We now claim that that $(\rho / 2,-\varepsilon \psi)$ is a subsolution of $\left(\mathrm{EP}_{\beta}\right)$ if $\beta$ is sufficiently large. To this end, we set $g(r):=\beta c\langle r\rangle^{-\eta}-C\langle r\rangle^{-1}$ for $r \geq 0$, where $\langle r\rangle:=\left(1+r^{2}\right)^{1 / 2}$. Suppose first that $0<\eta \leq 1$. Then $g \geq 0$ in $[0, \infty)$ for all $\beta \geq C / c$. This and (6.1) imply that $(\rho / 2,-\varepsilon \psi)$ is a subsolution of $\left(\mathrm{EP}_{\beta}\right)$ for any $\beta \geq C / c$. Suppose next that $\eta>1$. Then, by direct computations, we see that $g^{\prime}(r)=\langle r\rangle^{-3} r\left(C-\beta c \eta\langle r\rangle^{1-\eta}\right)$ for $r \geq 0$, and that $g$ attains its minimum in $[0, \infty)$ at $r^{*}$ such that $\left\langle r^{*}\right\rangle=(\eta \beta c / C)^{\frac{1}{\eta-1}}$ with minimum value $g\left(r^{*}\right)=-(\eta-1) c(C / \eta c)^{\frac{\eta}{\eta-1}} \beta^{-\frac{1}{\eta-1}}$. In particular, we have

$$
F[-\varepsilon \psi]-\beta V \leq-\rho-g(|x|) \leq-\rho+\nu \beta^{-\frac{1}{\eta-1}} \quad \text { in } \mathbf{R}^{d},
$$

where $\nu:=(\eta-1) c(C / \eta c)^{\frac{\eta}{\eta-1}}$. This implies that $(\rho / 2,-\varepsilon \psi)$ is a subsolution of $\left(\mathrm{EP}_{\beta}\right)$ if $\beta \geq$ $(2 \nu / \rho)^{\eta-1}$. Thus, in any case, we conclude that $(\rho / 2,-\varepsilon \psi)$ is a subsolution of $\left(\mathrm{EP}_{\beta}\right)$ if $\beta$ is sufficiently large. From this fact, we see that there exists a $\beta_{0}>0$ such that $\lambda_{\max }\left(\beta_{0}\right) \geq \rho / 2>0$. Hence, we have completed the proof.

The proof of Theorem 2.3 is now obvious from the above propositions.

In the rest of this section, we show that, contrary to the case where $\delta>0$ in (A1), the function $\beta \mapsto \lambda_{\max }(\beta)$ may not be strictly increasing if $\delta=0$.

Proposition 6.6. Let (A1) with $\delta=0$, (A2), and (A3c) hold. Assume the following:

(B) $b(x)=\rho \frac{x}{|x|}$ in $\mathbf{R}^{d} \backslash B_{R}$ for some $\rho, R>0, H(x, p)=\frac{1}{m}|p|^{m}$ in $\mathbf{R}^{d} \times \mathbf{R}^{d}$, and $0<\eta \leq 1$ in $(A 3 c)$.

Then, there exists a $\beta_{c}>0$ such that $\lambda_{\max }(\beta)=\lambda_{\max }\left(\beta_{c}\right)$ for all $\beta>\beta_{c}$.

Proof. We first prove that $\lambda_{\max }(\beta) \leq \rho^{m^{*}} / m^{*}$ for any $\beta>0$, where $m^{*}=m /(m-1)$. Let $\zeta \in$ $C^{\infty}\left(\mathbf{R}^{d}\right)$ be such that $\zeta \geq 0$ in $\mathbf{R}^{d}, \operatorname{supp} \zeta \subset B_{1}$, and $\int_{\mathbf{R}^{d}} \zeta(x)^{m^{*}} d x=1$. Let $(\lambda, \phi) \in \mathbf{R} \times C^{3}\left(\mathbf{R}^{d}\right)$ be any subsolution of $\left(\mathrm{EP}_{\beta}\right)$. Then, we see that

$$
\lambda+\frac{1}{2} \int_{\mathbf{R}^{d}} D\left(\zeta^{m^{*}}\right) \cdot D \phi d x+\int_{\mathbf{R}^{d}} \zeta^{m^{*}} b \cdot D \phi d x+\frac{1}{m} \int_{\mathbf{R}^{d}} \zeta^{m^{*}}|D \phi|^{m} d x \leq \beta \int_{\mathbf{R}^{d}} \zeta^{m^{*}} V d x .
$$

Fix any $l>m$. Then, by Young's inequality, there exists some $C(l)>0$ converging to $1 / m^{*}$ as $l \rightarrow m$ such that

$$
-\int_{\mathbf{R}^{d}} \zeta^{m^{*}} b \cdot D \phi d x \leq C(l) \int_{\mathbf{R}^{d}} \zeta^{m^{*}}|b|^{m^{*}} d x+\frac{1}{l} \int_{\mathbf{R}^{d}} \zeta^{m^{*}}|D \phi|^{m} d x .
$$

Setting $\nu:=(1 / m)-(1 / l)>0$, we observe that there exists a $C(\nu)>0$ such that

$$
\begin{aligned}
-\frac{1}{2} \int_{\mathbf{R}^{d}} D\left(\zeta^{m^{*}}\right) \cdot D \phi d x & =-\frac{m^{*}}{2} \int_{\mathbf{R}^{d}} \zeta^{m^{*}-1} D \zeta \cdot D \phi d x \\
& \leq C(\nu) \int_{\mathbf{R}^{d}}|D \zeta|^{m^{*}} d x+\nu \int_{\mathbf{R}^{d}} \zeta^{m^{*}}|D \phi|^{m} d x .
\end{aligned}
$$

Gathering these estimates, we obtain

$$
\lambda \leq C(\nu) \int_{\mathbf{R}^{d}}|D \zeta|^{m^{*}} d x+C(l) \int_{\mathbf{R}^{d}} \zeta^{m^{*}}|b|^{m^{*}} d x+\beta \int_{\mathbf{R}^{d}} \zeta^{m^{*}} V d x .
$$


Now, let $\varepsilon>0$ be arbitrary and set $\zeta_{\varepsilon}(x):=\varepsilon^{\frac{d}{m^{*}}} \zeta(\varepsilon x)$. Note that $\int_{\mathbf{R}^{d}} \zeta_{\varepsilon}(x)^{m^{*}} d x=1$ and $\int_{\mathbf{R}^{d}}\left|D \zeta_{\varepsilon}(x)\right|^{m^{*}} d x \leq C \varepsilon^{m^{*}}$ for some $C>0$ not depending on $\varepsilon$. Plugging $\zeta_{\varepsilon}$ into the above $\zeta$ and using the fact that $|b(x)|=\rho$ for all $x \in \mathbf{R}^{d} \backslash B_{R}$, we see that, for any $\theta>0$,

$$
\begin{aligned}
\lambda & \leq C(\nu) C \varepsilon^{m^{*}}+\int_{\mathbf{R}^{d}}\left(\mathbf{1}_{\mathbf{R}^{d} \backslash B_{R}}+\mathbf{1}_{B_{R}}\right) \zeta_{\varepsilon}^{m^{*}}|b|^{m^{*}} d x+\beta \int_{\mathbf{R}^{d}} \zeta_{\varepsilon}^{m^{*}}(V-\theta+\theta) d x \\
& \leq C(\nu) C \varepsilon^{m^{*}}+C(l) \rho^{m^{*}}+C(l) \sup _{B_{R}}|b|^{m^{*}} \int_{B_{R}} \zeta_{\varepsilon}^{m^{*}} d x+\beta \int_{\mathbf{R}^{d}} \zeta_{\varepsilon}^{m^{*}}(V-\theta)_{+} d x+\beta \theta .
\end{aligned}
$$

Since $\operatorname{supp}(V-\theta)_{+}$is compact and $\int_{K} \zeta_{\varepsilon}(x)^{m^{*}} d x=\int_{\varepsilon K} \zeta(y)^{m^{*}} d y \rightarrow 0$ as $\varepsilon \rightarrow 0$ for any compact subset $K \subset \mathbf{R}^{d}$, we see by sending $\varepsilon \rightarrow 0$ in the above inequality that $\lambda \leq C(l) \rho^{m^{*}}+\beta \theta$. Letting $l \rightarrow m$ and $\theta \rightarrow 0$, we obtain $\lambda \leq \rho^{m^{*}} / m^{*}$. This yields that $\lambda_{\max }(\beta) \leq \rho^{m^{*}} / m^{*}$.

We next prove that $\lambda_{\max }(\beta) \geq \rho^{m^{*}} / m^{*}$ for any sufficiently large $\beta$. We choose a $\psi \in C^{3}\left(\mathbf{R}^{d}\right)$ such that $\psi(x)=|x|$ in $\mathbf{R}^{d} \backslash B_{R}$. This is possible by setting $\psi(x):=f(|x|)$ in $B_{R}$ with $f(t)=$ $a_{1} t^{6}+a_{2} t^{4}+a_{3} t^{2}+a_{4}$ for $0 \leq t \leq 1$ and adjusting the coefficients so that $\psi \in C^{3}\left(\mathbf{R}^{d}\right)$. Since $D \psi(x)=|x|^{-1} x$ and $\Delta \psi(x)=(d-1)|x|^{-1}$ in $\mathbf{R}^{d} \backslash B_{R}$, we observe that, for any $x \in \mathbf{R}^{d} \backslash B_{R}$,

$$
F[-\varepsilon \psi](x)=\frac{\varepsilon}{2}(d-1)|x|^{-1}-\rho \varepsilon+\frac{\varepsilon^{m}}{m} .
$$

We now choose $\varepsilon$ as the minimum point of the function $\varepsilon \mapsto \varepsilon^{m} / m-\rho \varepsilon$, namely, we set $\varepsilon:=$ $\rho^{\frac{1}{m-1}}=\rho^{m^{*}-1}$. Then, $\varepsilon^{m} / m-\rho \varepsilon=-\rho^{m^{*}} / m^{*}$. Furthermore, since $0<\eta \leq 1$, we have

$$
\frac{\rho^{m^{*}}}{m^{*}}+F[-\varepsilon \psi]-\beta V \leq\left\{\frac{\rho^{m^{*}-1}}{2}(d-1)-\beta c\right\}|x|^{-1} \quad \text { in } \quad \mathbf{R}^{d} \backslash B_{R}
$$

for some $c>0$. The right-hand side of (6.2) is less than zero if $\beta>\beta_{1}:=\rho^{m^{*}-1}(d-1) /(2 c)$. On the other hand, since $\inf _{B_{R}} V>0$ by assumption, we see that $\rho^{m^{*}} / m^{*}+F[-\varepsilon \psi]-\beta V \leq 0$ in $B_{R}$ provided

$$
\beta>\beta_{2}:=\frac{\rho^{m^{*}} / m^{*}+\sup _{B_{R}} F[-\varepsilon \psi]}{\inf _{B_{R}} V} .
$$

In particular, $-\varepsilon \psi$ is a subsolution of (EP) if $\beta>\beta_{c}:=\max \left\{\beta_{1}, \beta_{2}\right\}$, which yields that $\lambda_{\max }(\beta) \geq$ $\rho^{m^{*}} / m^{*}$ for all $\beta>\beta_{c}$. Hence, we have completed the proof.

Remark 6.7. We do not know if Proposition 6.6 is valid without (B). In the proof of Proposition 6.6, we used the assumption that $0<\eta \leq 1$ to derive (6.2), only. We conjecture that, under (B) with $\eta>1$ instead of $0<\eta \leq 1$, the function $\beta \mapsto \lambda_{\max }(\beta)$ is strictly increasing in $[0, \infty)$.

Acknowledgment. NI is supported in part by JSPS KAKENHI Grant Number 15K04935. EC is partially supported by ANR-16-CE40-0015-01 (ANR project on Mean Field Games).

Appendix A: Gradient estimate for (EP). Let $\Omega \subset \mathbf{R}^{d}$ be a bounded domain with $C^{3}$ boundary. We consider the elliptic equation

$$
\varepsilon \phi-\frac{1}{2} \Delta \phi+b(x) \cdot D \phi+H(x, D \phi)-f=0 \text { in } \Omega,
$$

where $\varepsilon \geq 0$, and both $b: \Omega \rightarrow \mathbf{R}^{d}$ and $f: \Omega \rightarrow \mathbf{R}$ are of $C^{1}$-class. Furthermore, we assume that $H: \Omega \times \mathbf{R}^{d} \rightarrow \mathbf{R}$ satisfies the following:

(H) $H \in C^{1}\left(\Omega \times \mathbf{R}^{d} ; \mathbf{R}\right)$, and there exist some $m>1, \nu>0$ and $M>0$ such that

$$
H(x, p) \geq \nu|p|^{m}-M, \quad\left|D_{x} H(x, p)\right| \leq M\left(1+|p|^{m}\right), \quad\left|D_{p} H(x, p)\right| \leq M\left(1+|p|^{m-1}\right)
$$

for all $x \in \Omega$ and $p \in \mathbf{R}^{d}$.

TheOREm A.1. Let $(H)$ hold, and let $v, w \in C^{3}(D) \cap C(\bar{D})$ be, respectively, a subsolution and a supersolution of (A.1) such that $v \leq w$ in $\bar{D}$. Suppose either $\varepsilon>0$ or $v$ is a strict subsolution 
(i.e., strict inequality $<$ holds) in D. Then, there exists a solution $\phi \in C^{3}(D) \cap C(\bar{D})$ of $(A .1)$ such that $v \leq \phi \leq w$ in $\bar{D}$.

Proof. This theorem is a direct consequence of [27, Theorem 4.7.3] for $1<m \leq 2$, and [26, Théorème III-1] for $m>2$. $\square$

Theorem A.2. Let $(H)$ hold, and let $\Omega^{\prime}$ be a bounded domain in $\mathbf{R}^{d}$ with $C^{3}$ boundary such that $\Omega^{\prime} \subset \subset \Omega$. Then there exists a $K>0$ depending only on $d, m, \nu, M$, and $\operatorname{dist}\left(\Omega^{\prime}, \partial \Omega\right)$ such that, for any solution $\phi \in C^{3}(\Omega)$ of (A.1), the following estimate holds:

$$
\sup _{\Omega^{\prime}}|D \phi| \leq K\left[1+\sup _{\Omega}\left\{(\varepsilon \phi)_{-}^{\frac{1}{m}}+|b|^{\frac{1}{m-1}}+|D b|^{\frac{1}{2 m-2}}+f_{+}^{\frac{1}{m}}+|D f|^{\frac{1}{2 m-1}}\right\}\right],
$$

where $r_{ \pm}:=\max \{ \pm r, 0\}$ for $r \in \mathbf{R}$.

Proof. Fix any solution $\phi \in C^{3}(\Omega)$ of (A.1) and set $w:=(1 / 2)|D \phi|^{2}$. Then, we see that $D w=\left(D^{2} \phi\right)(D \phi)$ and

$$
\Delta w=\operatorname{tr}\left(\left(D^{2} \phi\right)^{2}\right)+D(\Delta \phi) \cdot D \phi
$$

where $D \phi$ and $D^{2} \phi$ denote the gradient vector and the Hessian matrix of $\phi$, respectively. In view of (A.1) and the Cauchy-Schwarz inequality, the first term of the right-hand side of (A.3) can be estimated as

$$
\begin{aligned}
d\left(\operatorname{tr}\left(D^{2} \phi\right)^{2}\right) & \geq\left(\operatorname{tr}\left(D^{2} \phi\right)\right)^{2}=(\Delta \phi)^{2}=4(\varepsilon \phi+b \cdot D \phi+H-f)^{2} \\
& =4\left\{H+(b \cdot D \phi+\varepsilon \phi-f)_{+}-(b \cdot D \phi+\varepsilon \phi-f)_{-}\right\}^{2} \\
& \geq 4\left\{H^{2}+(b \cdot D \phi+\varepsilon \phi-f)_{-}^{2}-2 H(b \cdot D \phi+\varepsilon \phi-f)_{-}\right\} \\
& \geq 2 H^{2}-4(b \cdot D \phi+\varepsilon \phi-f)_{-}^{2} \geq 2 H^{2}-4\left\{(b \cdot D \phi)_{-}+(\varepsilon \phi)_{-}+f_{+}\right\}^{2} \\
& \geq 2 H^{2}-12\left((b \cdot D \phi)_{-}^{2}+(\varepsilon \phi)_{-}^{2}+f_{+}^{2}\right) .
\end{aligned}
$$

On the other hand, by using (A.1) and (H), one can estimate the second term of the right-hand side of (A.3) as

$$
\begin{array}{rl}
D(\Delta \phi) \cdot D \phi= & 2 D(\varepsilon \phi+b \cdot D \phi+H-f) \cdot D \phi \\
= & 2 \varepsilon|D \phi|^{2}+2(D b)(D \phi) \cdot(D \phi)+2 b \cdot\left(D^{2} \phi\right)(D \phi) \\
& \quad+2 D_{x} H \cdot D \phi+2 D_{p} H \cdot\left(D^{2} \phi\right)(D \phi)-2 D f \cdot D \phi \\
\geq 2 & 2\left(b+D_{p} H\right) \cdot D w-2|D b||D \phi|^{2}-2 M\left(1+|D \phi|^{m}\right)|D \phi|-2|D f||D \phi| \\
\geq & 2\left(|b|+M+M|D \phi|^{m-1}\right)|D w|-2|D b||D \phi|^{2} \\
& \quad-2(M+|D f|)|D \phi|-2 M|D \phi|^{m+1},
\end{array}
$$

where $D b=\left(D_{i} b_{j}\right): \Omega \rightarrow \mathbf{R}^{d} \otimes \mathbf{R}^{d}$. Plugging these estimate into (A.3), we obtain

$$
\begin{aligned}
\Delta w \geq(2 / d) H^{2}-(12 / d)\left((\varepsilon \phi)_{-}^{2}+f_{+}^{2}\right)-\left\{(12 / d)|b|^{2}+2|D b|\right\}|D \phi|^{2} \\
\quad-2(M+|D f|)|D \phi|-2 M|D \phi|^{m+1}-2\left(|b|+M+M|D \phi|^{m-1}\right)|D w| .
\end{aligned}
$$

Now, let $\rho \in C^{2}(\Omega)$ be a cut-off function such that $\rho \equiv 1$ in $\Omega^{\prime}, \operatorname{supp} \rho \subset \Omega$, and $0 \leq \rho \leq 1$ in $\Omega$. Set $\eta:=\rho^{\gamma}$ with $\gamma=4 m /(m-1)$, and $z:=\eta w$. Let $x_{0}$ be a maximum point of $z$ on $\bar{\Omega}$. If $z\left(x_{0}\right)=0$, then there is nothing to prove. Hence, we assume that $z\left(x_{0}\right)>0$. Then, we see that $x_{0} \in \Omega$ since $z=0$ on $\partial \Omega$. In particular, $D z=\eta D w+w D \eta=0$ and $\Delta z \leq 0$ at $x=x_{0}$. Noting that $D w=-w(D \eta / \eta)=-(1 / 2)|D \phi|^{2}(D \eta / \eta)$ at $x=x_{0}$, the value of $\Delta z$ at $x=x_{0}$ can be evaluated as

$$
\begin{aligned}
0 \geq \Delta z & =\eta \Delta w+2 D \eta \cdot D w+w \Delta \eta=\eta \Delta w-|D \phi|^{2} \frac{|D \eta|^{2}}{\eta}+\frac{1}{2}|D \phi|^{2} \Delta \eta \\
& =\eta \Delta w-\left(\eta|D \phi|^{2 m}\right)^{\frac{1}{m}}\left(\eta^{-\frac{m+1}{2 m}}|D \eta|\right)^{2}+\frac{1}{2}\left(\eta|D \phi|^{2 m}\right)^{\frac{1}{m}}\left(\eta^{-\frac{1}{m}} \Delta \eta\right) .
\end{aligned}
$$


Furthermore, since $0 \leq \eta \leq \eta^{\frac{m+1}{2 m}} \leq \eta^{\frac{1}{m}} \leq \eta^{\frac{1}{2 m}} \leq 1$ and $2 \eta|D w|=|D \phi|^{2}|D \eta|$, we see in view of (A.4) and (H) that

$$
\begin{aligned}
\eta \Delta w \geq \frac{2}{d} & \eta\left(\nu|D \phi|^{m}-M\right)^{2}-\frac{12}{d} \eta\left((\varepsilon \phi)_{-}^{2}+f_{+}^{2}\right)-\left(\frac{12}{d}|b|^{2}+2|D b|\right)\left(\eta|D \phi|^{2 m}\right)^{\frac{1}{m}} \\
& -2(M+|D f|)\left(\eta|D \phi|^{2 m}\right)^{\frac{1}{2 m}}-2 M\left(\eta|D \phi|^{2 m}\right)^{\frac{m+1}{2 m}} \\
& -\left(|b|+M+M|D \phi|^{m-1}\right)|D \phi|^{2}|D \eta| .
\end{aligned}
$$

Plugging this into the previous inequality and setting $Z=\eta\left(x_{0}\right)\left|D \phi\left(x_{0}\right)\right|^{2 m}$, we obtain

$$
\begin{aligned}
0 \geq \frac{1}{d} & \left(\nu^{2} Z-M^{2}\right)-\frac{12}{d}\left((\varepsilon \phi)_{-}^{2}+f_{+}^{2}\right)-\left(\frac{12}{d}|b|^{2}+2|D b|\right) Z^{\frac{1}{m}}-2(M+|D f|) Z^{\frac{1}{2 m}} \\
& -2 M Z^{\frac{m+1}{2 m}}-(|b|+M)\left(\eta^{-\frac{1}{m}}|D \eta|\right) Z^{\frac{1}{m}}+M\left(\eta^{-\frac{m+1}{2 m}}|D \eta|\right) Z^{\frac{m+1}{2 m}} \\
& -\left(\eta^{-\frac{m+1}{2 m}}|D \eta|\right)^{2} Z^{\frac{1}{m}}-\left(\eta^{-\frac{1}{m}}|\Delta \eta|\right) Z^{\frac{1}{m}}
\end{aligned}
$$

Now, we apply Young's inequality to see that, for any $L>0$ and $\varepsilon>0$,

$$
L Z^{\frac{1}{2 m}} \leq \varepsilon Z+C_{\varepsilon} L^{\frac{2 m}{2 m-1}}, \quad L Z^{\frac{1}{m}} \leq \varepsilon Z+C_{\varepsilon} L^{\frac{m}{m-1}}, \quad L Z^{\frac{m+1}{2 m}} \leq \varepsilon Z+C_{\varepsilon} L^{\frac{2 m}{m-1}}
$$

where $C_{\varepsilon}>0$ is a constant depending only on $\varepsilon$ and $m$. Then, we have

$$
\begin{aligned}
Z \leq & K\left\{1+(\varepsilon \phi)_{-}^{2}+f_{+}^{2}+\left(|b|^{2}+|D b|\right)^{\frac{m}{m-1}}+|D f|^{\frac{2 m}{2 m-1}}\right. \\
& \left.+\left(1+|b|^{\frac{m}{m-1}}\right)\left(\eta^{-\frac{1}{m}}|D \eta|\right)^{\frac{m}{m-1}}+\left(\eta^{-\frac{m+1}{2 m}}|D \eta|\right)^{\frac{2 m}{m-1}}+\left(\eta^{-\frac{1}{m}}|\Delta \eta|\right)^{\frac{m}{m-1}}\right\} \\
\leq & K\left\{1+(\varepsilon \phi)_{-}^{2}+f_{+}^{2}+|b|^{\frac{2 m}{m-1}}+|D b|^{\frac{m}{m-1}}+|D f|^{\frac{2 m}{2 m-1}}\right. \\
& \left.+\left(\eta^{-\frac{1}{m}}|D \eta|\right)^{\frac{m}{m-1}}+\left(\eta^{-\frac{1}{m}}|D \eta|\right)^{\frac{2 m}{m-1}}+\left(\eta^{-\frac{m+1}{2 m}}|D \eta|\right)^{\frac{2 m}{m-1}}+\left(\eta^{-\frac{1}{m}}|\Delta \eta|\right)^{\frac{m}{m-1}}\right\}
\end{aligned}
$$

for some $K>0$ depending only on $d, \nu, M$, and $m$.

We now claim that $\eta^{-\frac{1}{m}}|D \eta|, \eta^{-\frac{m+1}{2 m}}|D \eta|$, and $\eta^{-\frac{1}{m}}|\Delta \eta|$ are bounded on $\Omega$ by a constant depending only on $m$ and $\operatorname{dist}\left(\Omega^{\prime}, \partial \Omega\right)$. Since $\eta^{\frac{m+1}{2 m}}<\eta^{\frac{1}{m}}$, it suffices to prove that $\eta^{-\theta}|D \eta|$ and $\eta^{-\theta}|\Delta \eta|$, with $\theta=\frac{m+1}{2 m}$, are bounded on $\Omega$. By direct computations, one can easily see that $\eta=\rho^{\gamma}$ satisfies

$$
\begin{aligned}
& \eta^{-\theta}|D \eta|=\gamma \rho^{\gamma-1-\gamma \theta}|D \rho|=\gamma \rho|D \rho|, \\
& \eta^{-\theta}|\Delta \eta| \leq \gamma\left\{\rho^{\gamma-1-\gamma \theta}|\Delta \rho|+(\gamma-1) \rho^{\gamma-2-\gamma \theta}|D \rho|^{2}\right\}=\gamma\left\{\rho|\Delta \rho|+(\gamma-1)|D \rho|^{2}\right\}
\end{aligned}
$$

where we have used the fact that $\gamma=4 m /(m-1)$ satisfies $\gamma-1-\gamma \theta=1$.

Hence, there exists some $K>0$ depending only on $d, \nu, M, m$, and $\operatorname{dist}\left(\Omega^{\prime}, \partial \Omega\right)$ such that, at $x=x_{0}$,

$$
\begin{aligned}
Z=\eta|D \phi|^{2 m} & \leq K\left(1+(\varepsilon \phi)_{-}^{2}+f_{+}^{2}+|b|^{\frac{2 m}{m-1}}+|D b|^{\frac{m}{m-1}}+|D f|^{\frac{2 m}{2 m-1}}\right) \\
& \leq K\left[1+\sup _{\Omega}\left\{(\varepsilon \phi)_{-}^{2}+f_{+}^{2}+|b|^{\frac{2 m}{m-1}}+|D b|^{\frac{m}{m-1}}+|D f|^{\frac{2 m}{2 m-1}}\right\}\right] .
\end{aligned}
$$

Since $\eta \equiv 1$ in $\Omega^{\prime}$, we see that

$$
\begin{aligned}
\sup _{x \in \Omega^{\prime}}|D \phi(x)|^{2} & =2 \sup _{x \in \Omega^{\prime}} \eta(x) w(x)=2 \sup _{x \in \Omega^{\prime}} z(x) \leq 2 \max _{x \in \bar{\Omega}} z(x)=2 z\left(x_{0}\right) \\
& =2 \eta\left(x_{0}\right) w\left(x_{0}\right)=\eta\left(x_{0}\right)^{\frac{m-1}{m}}\left(\eta\left(x_{0}\right)\left|D \phi\left(x_{0}\right)\right|^{2 m}\right)^{\frac{1}{m}} \leq Z^{\frac{1}{m}},
\end{aligned}
$$

which implies the desired estimate. Hence, we have completed the proof. 
Appendix B: Moment estimates for the solution of (1.3). We first recall a moment estimate for the controlled process $X^{\xi}=\left(X_{t}^{\xi}\right)_{t \geq 0}$ governed by (1.3). We notice that $\left\langle X_{t}^{\xi}\right\rangle=$ $\left(1+\left|X_{t}^{\xi}\right|^{2}\right)^{1 / 2}$, which should not be confused with the quadratic variation of $X^{\xi}$.

Lemma B.1. Let (A1) hold. Then there exists a $C>0$ such that for any $\xi \in \mathcal{A}, x \in \mathbf{R}^{d}$, and $T>0$,

$$
\mathbf{E}_{x}\left[\sup _{0 \leq t \leq T}\left\langle X_{t}^{\xi}\right\rangle^{1+\frac{\delta}{m-1}}\right] \leq C\left(\langle x\rangle^{1+\frac{\delta}{m-1}}+T+\mathbf{E}_{x}\left[\int_{0}^{T}\left|\xi_{t}\right|^{m^{*}} d t\right]\right) .
$$

Moreover, if $\phi \in C^{2}\left(\mathbf{R}^{d}\right)$ satisfies $|D \phi(x)| \leq C\langle x\rangle^{\frac{\delta}{m-1}}$ in $\mathbf{R}^{d}$ for some $C>0$, then the stochastic integral $\int_{0}^{T} D \phi\left(X_{t}^{\xi}\right) d W_{t}$ is integrable with respect to $\mathbf{P}_{x}$ for all $x \in \mathbf{R}^{d}$.

Proof. For simplicity, we set $\gamma:=1+\frac{\delta}{m-1}$. We apply Ito's formula to $\gamma^{-1}\left\langle X_{t}^{\xi}\right\rangle^{\gamma}$. Then, since $D\left(\gamma^{-1}\langle x\rangle^{\gamma}\right)=\langle x\rangle^{\gamma-2} x$ and $\Delta\left(\gamma^{-1}\langle x\rangle^{\gamma}\right) \leq(|\gamma-2|+d)\langle x\rangle^{\gamma-2}$ in $\mathbf{R}^{d}$, we have

$$
\begin{aligned}
\frac{1}{\gamma}\left\langle X_{T \wedge \tau_{n}}^{\xi}\right\rangle^{\gamma} \leq & \frac{1}{\gamma}\langle x\rangle^{\gamma}+(|\gamma-2|+d) \int_{0}^{T \wedge \tau_{n}}\left\langle X_{t}^{\xi}\right\rangle^{\gamma-2} d t-\int_{0}^{T \wedge \tau_{n}} \xi_{t} \cdot\left(\left\langle X_{t}^{\xi}\right\rangle^{\gamma-2} X_{t}^{\xi}\right) d t \\
& -\int_{0}^{T \wedge \tau_{n}}\left\langle X_{t}^{\xi}\right\rangle^{\gamma-2}\left(b\left(X_{t}^{\xi}\right) \cdot X_{t}^{\xi}\right) d t+\left|\int_{0}^{T \wedge \tau_{n}}\left\langle X_{t}^{\xi}\right\rangle^{\gamma-2} X_{t}^{\xi} d W_{t}\right|
\end{aligned}
$$

for any $n \geq 1$, where $\tau_{n}:=\inf \left\{t>0|| X_{t}^{\xi} \mid>n\right\}$. In view of (A1), there exist some $\nu, K>0$ such that the fourth term of the right-hand side can be estimated as

$$
\begin{aligned}
-\int_{0}^{T \wedge \tau_{n}}\left\langle X_{t}^{\xi}\right\rangle^{\gamma-2}\left(b\left(X_{t}^{\xi}\right) \cdot X_{t}^{\xi}\right) d t & \leq-\int_{0}^{T \wedge \tau_{n}}\left\langle X_{t}^{\xi}\right\rangle^{\gamma-2}\left(\nu\left\langle X_{t}^{\xi}\right\rangle^{1+\delta}-K\right) d t \\
& =-\nu \int_{0}^{T \wedge \tau_{n}}\left\langle X_{t}^{\xi}\right\rangle^{\gamma-1+\delta} d t+K \int_{0}^{T \wedge \tau_{n}}\left\langle X_{t}^{\xi}\right\rangle^{\gamma-2} d t .
\end{aligned}
$$

Since $\gamma-2<m^{*} \delta$, there exists some constant $C>0$ depending only on $d, \delta, m$, and the above $\nu$, $K$ such that

$$
(|\gamma-2|+d+K) \int_{0}^{T \wedge \tau_{n}}\left\langle X_{t}^{\xi}\right\rangle^{\gamma-2} d t \leq \frac{\nu}{2} \int_{0}^{T \wedge \tau_{n}}\left\langle X_{t}^{\xi}\right\rangle^{\gamma-1+\delta} d t+C\left(T \wedge \tau_{n}\right) .
$$

In what follows, $C>0$ denotes various constants depending only on $d, \delta, m$, and the above $\nu, K$. Applying Young's inequality, we also have

$$
\begin{aligned}
-\int_{0}^{T \wedge \tau_{n}} \xi_{t} \cdot\left(\left\langle X_{t}^{\xi}\right\rangle^{\gamma-2} X_{t}^{\xi}\right) d t & \leq \int_{0}^{T \wedge \tau_{n}}\left(C\left|\xi_{t}\right|^{m^{*}}+\frac{\nu}{4}\left|\left\langle X_{t}^{\xi}\right\rangle^{\gamma-2} X_{t}^{\xi}\right|^{m}\right) d t \\
& \leq C \int_{0}^{T \wedge \tau_{n}}\left|\xi_{t}\right|^{m^{*}} d t+\frac{\nu}{4} \int_{0}^{T \wedge \tau_{n}}\left\langle X_{t}^{\xi}\right\rangle^{m(\gamma-1)} d t .
\end{aligned}
$$

Since $\gamma-1+\delta=m(\gamma-1)=m^{*} \delta$, we conclude that

$$
\begin{aligned}
& \frac{1}{\gamma} \mathbf{E}_{x}\left[\sup _{0 \leq t \leq T}\left\langle X_{t \wedge \tau_{n}}^{\xi}\right\rangle^{\gamma}\right]+\frac{\nu}{4} \mathbf{E}_{x}\left[\int_{0}^{T \wedge \tau_{n}}\left\langle X_{t}^{\xi}\right\rangle^{m^{*} \delta} d t\right] \\
& \leq \frac{1}{\gamma}\langle x\rangle^{\gamma}+C T+C \mathbf{E}_{x}\left[\int_{0}^{T \wedge \tau_{n}}\left|\xi_{t}\right|^{m^{*}} d t\right]+\mathbf{E}_{x}\left[\sup _{0 \leq t \leq T}\left|\int_{0}^{T \wedge \tau_{n}}\left\langle X_{t}^{\xi}\right\rangle^{\gamma-2} X_{t}^{\xi} d W_{t}\right|\right] .
\end{aligned}
$$

We now use the Burkholder-Davis-Gundy inequality to obtain

$$
\mathbf{E}_{x}\left[\sup _{0 \leq t \leq T}\left|\int_{0}^{T \wedge \tau_{n}}\left\langle X_{t}^{\xi}\right\rangle^{\gamma-2} X_{t}^{\xi} d W_{t}\right|\right] \leq C \mathbf{E}_{x}\left[\left(\int_{0}^{T \wedge \tau_{n}}\left\langle X_{t}^{\xi}\right\rangle^{2(\gamma-1)} d t\right)^{\frac{1}{2}}\right] .
$$


Making use of Young's inequality twice, we see that, for any $\varepsilon_{1}, \varepsilon_{2}>0$, there exists a $C_{\varepsilon}>0$ such that

$$
\begin{gathered}
\mathbf{E}_{x}\left[\left(\int_{0}^{T \wedge \tau_{n}}\left\langle X_{t}^{\xi}\right\rangle^{2(\gamma-1)} d t\right)^{\frac{1}{2}}\right] \leq \mathbf{E}_{x}\left[\left(\sup _{0 \leq t \leq T \wedge \tau_{n}}\left\langle X_{t}^{\xi}\right\rangle^{\frac{\gamma-1}{2}}\right)\left(\int_{0}^{T \wedge \tau_{n}}\left\langle X_{t}^{\xi}\right\rangle^{\gamma-1} d t\right)^{\frac{1}{2}}\right] \\
\quad \leq \varepsilon_{1} \mathbf{E}_{x}\left[\sup _{0 \leq t \leq T \wedge \tau_{n}}\left\langle X_{t}^{\xi}\right\rangle^{\gamma-1}\right]+\varepsilon_{2} \mathbf{E}_{x}\left[\int_{0}^{T \wedge \tau_{n}}\left\langle X_{t}^{\xi}\right\rangle^{m(\gamma-1)} d t\right]+C_{\varepsilon} \mathbf{E}_{x}\left[T \wedge \tau_{n}\right] .
\end{gathered}
$$

Choosing $\varepsilon_{1}, \varepsilon_{2}$ sufficiently small, we finally obtain the estimate of the form

$$
\mathbf{E}_{x}\left[\sup _{0 \leq t \leq T \wedge \tau_{n}}\left\langle X_{t}^{\xi}\right\rangle^{\gamma}\right] \leq C\langle x\rangle^{\gamma}+C T+C \mathbf{E}_{x}\left[\int_{0}^{T \wedge \tau_{n}}\left|\xi_{t}\right|^{m^{*}} d t\right] .
$$

Letting $n \rightarrow \infty$ in the above inequality, we have the desired estimate.

In order to prove the latter claim, we observe by the Burkholder-Davis-Gundy inequality that

$$
\mathbf{E}_{x}\left[\left|\int_{0}^{T} D \phi\left(X_{t}^{\xi}\right) d W_{t}\right|\right] \leq C \mathbf{E}_{x}\left[\left(\int_{0}^{T}\left|D \phi\left(X_{t}^{\xi}\right)\right|^{2} d t\right)^{\frac{1}{2}}\right] \leq C T \mathbf{E}_{x}\left[\sup _{0 \leq t \leq T}\left\langle X_{t}^{\xi}\right\rangle^{\frac{\delta}{m-1}}\right] .
$$

Since $\frac{\delta}{m-1} \leq m^{*} \delta \leq 1+\frac{\delta}{m-1}$, we conclude from the previous estimate that the right-hand side is finite. Hence, we have completed the proof. $\square$

Lemma B.2. Let (A1), (A2), and (A3a) hold. Let $(\lambda, \phi) \in \mathbf{R} \times C^{3}\left(\mathbf{R}^{d}\right)$ be a subsolution of (EP). Then, for any $\xi \in \mathcal{A}$,

$$
\mathbf{E}_{x}\left[\int_{0}^{T}\left(L\left(X_{t}^{\xi}, \xi\right)+V\left(X_{t}^{\xi}\right)\right) d t\right] \geq \lambda T+\phi(x)-\mathbf{E}_{x}\left[\phi\left(X_{T}^{\xi}\right)\right], \quad x \in \mathbf{R}^{d}, \quad T>0 .
$$

Moreover, the above inequality holds with equality if $(\lambda, \phi) \in \mathbf{R} \times C^{3}\left(\mathbf{R}^{d}\right)$ is a solution of (EP) and the control process $\xi^{*}=\left(\xi_{t}^{*}\right)_{t \geq 0}$ defined by $\xi_{t}^{*}:=D_{p} H\left(X_{t}, D \phi\left(X_{t}\right)\right), t \geq 0$, belongs to $\mathcal{A}$, where $X=\left(X_{t}\right)_{t \geq 0}$ stands for the associated $A^{\phi}$-diffusion.

Proof. We apply Ito's formula to $\phi\left(X_{t}^{\xi}\right)$. Then, since $|D \phi(x)| \leq C\langle x\rangle^{\frac{\delta}{m-1}}$ in $\mathbf{R}^{d}$ for some $C>0$ and $H(x, p)+L(x, \xi) \geq \xi \cdot p$ for all $x, p, \xi \in \mathbf{R}^{d}$, we see in view of the subsolution property of $(\lambda, \phi)$ and Lemma B.1 that

$$
\begin{aligned}
\mathbf{E}_{x}\left[\phi\left(X_{T}^{\xi}\right)\right]-\phi(x) & =\mathbf{E}_{x}\left[\int_{0}^{T}\left\{\frac{1}{2} \Delta \phi\left(X_{t}^{\xi}\right)-\left(b\left(X_{t}^{\xi}\right)+\xi_{t}\right) \cdot D \phi\left(X_{t}^{\xi}\right)\right\} d t\right] \\
& \geq \mathbf{E}_{x}\left[\int_{0}^{T}\left(\lambda+H\left(X_{t}^{\xi}, D \phi\left(X_{t}^{\xi}\right)\right)-V\left(X_{t}^{\xi}\right)-\xi_{t} \cdot D \phi\left(X_{t}^{\xi}\right)\right) d t\right] \\
& \geq \lambda T-\mathbf{E}_{x}\left[\int_{0}^{T}\left(L\left(X_{t}^{\xi}, \xi_{t}\right)+V\left(X_{t}^{\xi}\right)\right) d t\right],
\end{aligned}
$$

which is the former claim. In order to prove the latter claim, suppose that $(\lambda, \phi)$ is a solution of (EP) and set $\xi_{t}^{*}:=D_{p} H\left(X_{t}, D \phi\left(X_{t}\right)\right)$. Then, the first inequality can be replaced by equality, and the second inequality holds with equality in view of the equivalence $H(x, p)+L(x, \xi)=\xi \cdot p \Leftrightarrow$ $\xi=D_{p} H(x, p)$. Hence, we have completed the proof.

Appendix C: Comparison principle for (5.1). We characterize the value function $u(T, x)$ defined by (2.8) as the minimal solution, in a suitable class of functions, to the Cauchy problem (5.1). More precisely, let $\hat{\Psi}$ be the collection of functions $u \in C^{1,2}\left((0, \infty) \times \mathbf{R}^{d}\right) \cap C\left([0, \infty) \times \mathbf{R}^{d}\right)$ such that, for some $q>1$,

$$
\sup _{0 \leq t \leq T, x \in \mathbf{R}^{d}} \frac{u(t, x)}{\langle x\rangle^{q}}<\infty, \quad \inf _{0 \leq t \leq T} \liminf _{|x| \rightarrow \infty} \frac{u(t, x)}{\langle x\rangle^{1+\frac{\delta}{m-1}}} \geq 0, \quad T>0 .
$$


Then, one has the following.

Theorem C.1. Let (A1), (A2), and (A3a) hold. Suppose that $g \in \Psi_{1+\frac{\delta}{m-1}}$. Then the following (a)-(c) are valid:

(a) For any subsolution $v \in C^{1,2}\left((0, \infty) \times \mathbf{R}^{d}\right) \cap C\left([0, \infty) \times \mathbf{R}^{d}\right)$ of (5.1) which satisfies

$\sup _{0 \leq t \leq T, x \in \mathbf{R}^{d}}|v(t, x)|\langle x\rangle^{-\left(1+\frac{\delta}{m-1}\right)}<\infty$ for all $T>0$,

$$
v(T+S, x) \leq \inf _{\xi \in \mathcal{A}} \mathbf{E}_{x}\left[\int_{0}^{T}\left(L\left(X_{t}^{\xi}, \xi_{t}\right)+V\left(X_{t}^{\xi}\right)\right) d t+v\left(S, X_{T}^{\xi}\right)\right], \quad T, S \geq 0, \quad x \in \mathbf{R}^{d} .
$$

(b) For any supersolution $v \in \hat{\Psi}$ of (5.1),

$$
v(T+S, x) \geq \inf _{\xi \in \mathcal{A}} \mathbf{E}_{x}\left[\int_{0}^{T}\left(L\left(X_{t}^{\xi}, \xi_{t}\right)+V\left(X_{t}^{\xi}\right)\right) d t+v\left(S, X_{T}^{\xi}\right)\right], \quad T, S \geq 0, \quad x \in \mathbf{R}^{d} .
$$

(c) The value function u defined by (2.8) is the minimal solution of (5.1) in $\hat{\Psi}$. Moreover, for any $R>0$, there exists a $C_{R}>0$ such that $\sup _{t \geq 1, x \in B_{R}}|D u(t, x)| \leq C_{R}$.

Proof. We first show (a). Fix any $T, S \geq 0, x \in \mathbf{R}^{d}, \xi \in \mathcal{A}$, and apply Ito's formula to $v\left(T+S-t, X_{t}^{\xi}\right)$. Then, from the subsolution property of $v$, together with the inequality $H(x, p)+L(x, \xi) \geq \xi \cdot p$ for all $x, p, \xi \in \mathbf{R}^{d}$, we see that

$$
v(T+S, x) \leq \mathbf{E}_{x}\left[\int_{0}^{T \wedge \tau_{n}}\left(L\left(X_{t}^{\xi}, \xi_{t}\right)+V\left(X_{t}^{\xi}\right)\right) d t+v\left(T+S-T \wedge \tau_{n}, X_{T \wedge \tau_{n}}^{\xi}\right)\right]
$$

for all $n \geq 1$, where $\tau_{n}:=\inf \left\{t>0|| X_{t}^{\xi} \mid>n\right\}$. Since $|v(t, x)| \leq C\langle x\rangle^{1+\frac{\delta}{m-1}}$ in $[0, T+S] \times \mathbf{R}^{d}$ for some $C>0$, we see from Lemma B.1 that the family $\left\{v\left(T+S-T \wedge \tau_{n}, X_{T \wedge \tau_{n}}^{\xi}\right)\right\}_{n \geq 1}$ is uniformly integrable. Noting this and the fact that $L+V$ is bounded below in $\mathbf{R}^{d} \times \mathbf{R}^{d}$, we conclude by sending $n \rightarrow \infty$ in the above inequality that

$$
v(T+S, x) \leq \mathbf{E}_{x}\left[\int_{0}^{T}\left(L\left(X_{t}^{\xi}, \xi_{t}\right)+V\left(X_{t}^{\xi}\right)\right) d t+v\left(S, X_{T}^{\xi}\right)\right] .
$$

Since $\xi \in \mathcal{A}$ is arbitrary, we obtain the desired estimate.

We next prove (b). Let $X=\left(X_{t}\right)_{t \geq 0}$ be the diffusion process governed by

$$
d X_{t}=-b\left(X_{t}\right) d t-D_{p} H\left(X_{t}, D v\left(T+S-t, X_{t}\right)\right) d t+d W_{t},
$$

and set $p_{t}:=D v\left(T+S-t, X_{t}\right)$ and $\xi_{t}:=D_{p} H\left(X_{t}, p_{t}\right)$. We claim here that $X$ does not explode in finite time. To see this, we set $\psi(x):=-\varepsilon\langle x\rangle^{1+\frac{\delta}{m-1}}$, where we choose $\varepsilon>0$ so that $F[\psi] \leq$ $C-\rho\langle x\rangle^{m^{*} \delta}$ in $\mathbf{R}^{d}$ for some $C, \rho>0$ (see Proposition 4.4). We apply Ito's formula to $v\left(T+S-t, X_{t}\right.$ ). Then, by using the supersolution property of $v$ and the equality $H\left(X_{t}, p_{t}\right)+L\left(X_{t}, \xi_{t}\right)=\xi_{t} \cdot p_{t}$, we see that

$$
v(T+S, x) \geq \mathbf{E}_{x}\left[v\left(T+S-T \wedge \tau_{n}, X_{T \wedge \tau_{n}}\right)+\int_{0}^{T \wedge \tau_{n}}\left(L\left(X_{t}, \xi_{t}\right)+V\left(X_{t}\right)\right) d t\right]
$$

for all $n \geq 1$, where $\tau_{n}:=\inf \left\{t>0|| X_{t} \mid>n\right\}$. Furthermore, we apply Ito's formula to $\psi\left(X_{t}\right)$. Then, observing that $H\left(X_{t}, D \psi\left(X_{t}\right)\right)+L\left(X_{t}, \xi_{t}\right) \geq \xi_{t} \cdot D \psi\left(X_{t}\right)$, we have

$$
\begin{aligned}
\mathbf{E}_{x}\left[\psi\left(X_{T \wedge \tau_{n}}\right)\right]-\psi(x) & =\mathbf{E}_{x}\left[\int_{0}^{T \wedge \tau_{n}}\left(\frac{1}{2} \Delta \psi\left(X_{t}\right)-b\left(X_{t}\right) \cdot D \psi\left(X_{t}\right)-\xi_{t} \cdot D \psi\left(X_{t}\right)\right) d t\right] \\
& =\mathbf{E}_{x}\left[\int_{0}^{T \wedge \tau_{n}}\left(H\left(X_{t}, D \psi\left(X_{t}\right)\right)-F[\psi]\left(X_{t}\right)-\xi_{t} \cdot D \psi\left(X_{t}\right)\right) d t\right] .
\end{aligned}
$$


Adding both sides of the above two inequalities, we obtain

$$
(v-\psi)(T+S, x) \geq \mathbf{E}_{x}\left[(v-\psi)\left(T+S-T \wedge \tau_{n}, X_{T \wedge \tau_{n}}\right)+\int_{0}^{T \wedge \tau_{n}} f\left(X_{t}, \xi\right) d t\right]
$$

where we have set

$$
f(x, \xi):=H(x, D \psi(x))+L(x, \xi)-\xi \cdot D \psi(x)+V(x)-F[\psi](x)
$$

for $(x, \xi) \in \mathbf{R}^{d} \times \mathbf{R}^{d}$. Note that $f$ is bounded below in $\mathbf{R}^{d} \times \mathbf{R}^{d}$. Since $v \in \hat{\Psi}$, we observe that $\inf _{0 \leq t \leq T+S}(v-\psi)(t, x) \rightarrow \infty$ as $|x| \rightarrow \infty$. In particular, $v-\psi$ is bounded below in $[0, T+S] \times \mathbf{R}^{d}$. Thus, letting $n \rightarrow \infty$ in the above inequality, we have

$$
(v-\psi)(T+S, x) \geq \mathbf{E}_{x}\left[(v-\psi)\left(T+S-T \wedge \tau_{\infty}, X_{T \wedge \tau_{\infty}}\right)+\int_{0}^{T \wedge \tau_{\infty}} f\left(X_{t}, \xi\right) d t\right]
$$

where $\tau_{\infty}:=\lim _{n \rightarrow \infty} \tau_{n}$. This yields that $\mathbf{P}_{x}\left(\tau_{\infty}<\infty\right)=1$ for any $x \in \mathbf{R}^{d}$. Hence, $X$ does not explode in finite time. Noting that $v-\psi$ and $L+V$ are bounded below and that $\left\{\psi\left(X_{T \wedge \tau_{n}}\right)\right\}_{n \geq 1}$ is uniformly integrable, we conclude by sending $n \rightarrow \infty$ in (C.1) that

$$
\begin{aligned}
v(T+S, x) \geq \liminf _{n \rightarrow \infty} \mathbf{E}_{x}\left[(v-\psi)\left(T+S-T \wedge \tau_{n}, X_{T \wedge \tau_{n}}\right)+\psi\left(X_{T \wedge \tau_{n}}\right)\right] \\
\quad+\lim _{n \rightarrow \infty} \mathbf{E}_{x}\left[\int_{0}^{T \wedge \tau_{n}}\left(L\left(X_{t}, \xi_{t}\right)+V\left(X_{t}\right)\right) d t\right] \\
=\mathbf{E}_{x}\left[v\left(S, X_{T}\right)+\int_{0}^{T}\left(L\left(X_{t}, \xi_{t}\right)+V\left(X_{t}\right)\right) d t\right] \\
\geq \inf _{\xi \in \mathcal{A}} \mathbf{E}_{x}\left[v\left(S, X_{T}^{\xi}\right)+\int_{0}^{T}\left(L\left(X_{t}^{\xi}, \xi_{t}\right)+V\left(X_{t}^{\xi}\right)\right) d t\right] .
\end{aligned}
$$

We finally prove (c). Let $\psi$ be as above. Since $(g-\psi)(x) \rightarrow \infty$ as $|x| \rightarrow \infty$, we may assume, by adding a constant to $g$ if necessary, that $g \geq \psi$ in $\mathbf{R}^{d}$.

We first show that there exists a solution $\hat{u} \in C^{1,2}\left((0, \infty) \times \mathbf{R}^{d}\right) \cap C\left([0, \infty) \times \mathbf{R}^{d}\right)$ of (5.1) such that $\hat{u} \leq u$. Let $\left\{g_{n}^{(1)}\right\}$ and $\left\{g_{n}^{(2)}\right\}$ be two sequences of bounded and nonnegative smooth functions on $\mathbf{R}^{d}$ that are non-decreasing with resect to $n$, and $g_{n}^{(1)} \rightarrow g-\psi$ and $g_{n}^{(2)} \rightarrow-\psi$ in $C\left(\mathbf{R}^{d}\right)$ as $n \rightarrow \infty$. For $n \geq 1$, we set $g_{n}:=g_{n}^{(1)}-g_{n}^{(2)}$ and define $u_{n}:[0, \infty) \times \mathbf{R}^{d} \rightarrow \mathbf{R}$ by

$$
u_{n}(T, x):=\inf _{\xi \in \mathcal{A}} \mathbf{E}_{x}\left[\int_{0}^{T}\left(L\left(X_{t}^{\xi}, \xi_{t}\right)+V\left(X_{t}^{\xi}\right)\right) d t+g_{n}\left(X_{T}^{\xi}\right)\right], \quad T \geq 0, \quad x \in \mathbf{R}^{d}
$$

Then, it is known (e.g., [13, Theorem IV.11.1, Remark IV.11.2]) that $u_{n} \in C^{1,2}\left((0, \infty) \times \mathbf{R}^{d}\right) \cap$ $C\left([0, \infty) \times \mathbf{R}^{d}\right)$ and $u_{n}$ is a solution to (5.1) with initial function $g_{n}$ in place of $g$. Moreover, similarly as in the proof of Theorem A.2 (see also [18, Theorem 3.2]), one can verify that, for any $R>0$ and $\delta>0$, there exists a $K>0$ such that

$$
\sup _{n \geq 1} \sup _{(t, x) \in[2 \delta, \infty) \times B_{R}}\left|D u_{n}(t, x)\right| \leq K .
$$

This and the classical regularity estimate imply that $\left\{u_{n}\right\}$ is precompact in $C^{1,2}\left((0, \infty) \times \mathbf{R}^{d}\right)$. Since $\left\{g_{n}\right\}$ converges to $g$ in $C\left(\mathbf{R}^{d}\right)$ as $n \rightarrow \infty$, we see that, along a suitable subsequence, $\left\{u_{n}\right\}$ converges to a function $\hat{u} \in C^{1,2}\left((0, \infty) \times \mathbf{R}^{d}\right) \cap C\left([0, \infty) \times \mathbf{R}^{d}\right)$, and $\hat{u}$ is a solution of (5.1). 
The inequality $\hat{u} \leq u$ can be verified as follows. By the definitions of $g_{n}$ and $u_{n}$, we see that, for any $T \geq 0, x \in \mathbf{R}^{d}$, and $\xi \in \mathcal{A}$,

$$
\begin{aligned}
u_{n}(T, x) & \leq \mathbf{E}_{x}\left[\int_{0}^{T}\left(L\left(X_{t}^{\xi}, \xi_{t}\right)+V\left(X_{t}^{\xi}\right)\right) d t+g_{n}\left(X_{T}^{\xi}\right)\right] \\
& =\mathbf{E}_{x}\left[\int_{0}^{T}\left(L\left(X_{t}^{\xi}, \xi_{t}\right)+V\left(X_{t}^{\xi}\right)\right) d t+g_{n}^{(1)}\left(X_{T}^{\xi}\right)-g_{n}^{(2)}\left(X_{T}^{\xi}\right)\right] .
\end{aligned}
$$

Since $0 \leq \mathbf{E}_{x}\left[(-\psi)\left(X_{T}^{\xi}\right)\right]<\infty$ for all $x \in \mathcal{A}$ by Lemma B.1, we conclude by letting $n \rightarrow \infty$ and using the monotone convergence theorem in the above inequality that

$$
\hat{u}(T, x) \leq \mathbf{E}_{x}\left[\int_{0}^{T}\left(L\left(X_{t}^{\xi}, \xi_{t}\right)+V\left(X_{t}^{\xi}\right)\right) d t+g\left(X_{T}^{\xi}\right)\right] .
$$

Taking the infimum over all $\xi \in \mathcal{A}$, we obtain $\hat{u} \leq u$.

We next prove that $\hat{u} \in \hat{\Psi}$. We first check that $\inf _{[0, T] \times \mathbf{R}^{d}}(\hat{u}(t, x)-\psi)>-\infty$ for any $T>0$. By the definition of $g_{n}$, we see that $g_{n}=g_{n}^{(1)}-g_{n}^{(2)} \geq-g_{n}^{(2)} \geq \psi$ in $\mathbf{R}^{d}$. Thus,

$$
u_{n}(T, x) \geq \inf _{\xi \in \mathcal{A}} \mathbf{E}_{x}\left[\int_{0}^{T}\left(L\left(X_{t}^{\xi}, \xi_{t}\right)+V\left(X_{t}^{\xi}\right)\right) d t+\psi\left(X_{T}^{\xi}\right)\right], \quad T \geq 0, \quad x \in \mathbf{R}^{d} .
$$

Similarly as in the proof of (b), we observe that, for any $\xi \in \mathcal{A}$,

$$
\mathbf{E}_{x}\left[\psi\left(X_{T}^{\xi}\right)\right] \geq \psi(x)+\mathbf{E}_{x}\left[\int_{0}^{T}\left(-F[\psi]\left(X_{t}^{\xi}\right)-L\left(X_{t}^{\xi}, \xi\right)\right) d t\right] .
$$

Plugging this into the previous inequality, we obtain

$$
u_{n}(T, x)-\psi(x) \geq \inf _{\xi \in \mathcal{A}} \mathbf{E}_{x}\left[\int_{0}^{T}\left(V\left(X_{t}^{\xi}\right)-F[\psi]\left(X_{t}^{\xi}\right)\right) d t\right] \geq-C T
$$

for some $C>0$ not depending on $n$ and $(T, x)$. Letting $n \rightarrow \infty$, we conclude that $\hat{u}-\psi \geq-C$ in $\mathbf{R}^{d}$. We now show that there exists some $q>1$ such that, for any $T>0, u(t, x) \leq C\langle x\rangle^{q}$ in $[0, T] \times \mathbf{R}^{d}$ for some $C>0$. To see this, let $X=\left(X_{t}\right)_{t \geq 0}$ be the $A^{0}$-diffusion, namely, the solution of (1.3) with $\xi_{t} \equiv 0$. Then, since $L(x, 0)=0, V \leq C$, and $g_{n} \leq g \leq C\langle x\rangle^{q}$ in $\mathbf{R}^{d}$ for some $C>0$ and $q>1$, we see that

$$
u_{n}(T, x) \leq \mathbf{E}_{x}\left[\int_{0}^{T}\left(L\left(X_{t}, 0\right)+V\left(X_{t}\right)\right) d t+g_{n}\left(X_{T}\right)\right] \leq C T+C \mathbf{E}_{x}\left[\left\langle X_{T}\right\rangle^{q}\right] .
$$

Since $\mathbf{E}_{x}\left[\left\langle X_{T}\right\rangle^{q}\right] \leq K\left(\langle x\rangle^{q}+1\right)$ in $\mathbf{R}^{d}$ for some $K>0$, we conclude by sending $n \rightarrow \infty$ that $\sup _{(t, x) \in[0, T] \times \mathbf{R}^{d}}\left(\hat{u}(t, x) /\langle x\rangle^{q}\right)<\infty$ for all $T>0$. Hence, $\hat{u} \in \hat{\Psi}$.

The rest of the proof is now easy. Indeed, since $\hat{u}$ is a solution of (5.1) which belongs to $\hat{\Psi}$, we have $u \leq \hat{u}$ in view of (b). The opposite inequality $\hat{u} \leq u$ has been proved. Hence, $\hat{u}=u$, so that $u \in \hat{\Psi}$. The minimality of $u$ is also obvious from (b). The gradient estimate comes directly from (C.2). Hence, the proof of (c) is complete.

\section{REFERENCES}

[1] A. Arapostathis, V. S. Borkar, M. K. Ghosh, Ergodic Control of Diffusion Processes, Cambridge University Press, New York, 2012.

[2] A. Arapostathis, V. S. Borkar, K.S. Kumar, Convergence of the relative value iteration for the ergodic control problem of nondegenerate diffusions under near-monotone costs, SIAM J. Control Optim., 52 (2014), pp. $1-31$. 
[3] A. Arapostathis, A. Biswas, S. Saha, Strict monotonicity of principal eigenvalues of elliptic operators in $\mathbb{R}^{d}$ and risk-sensitive control, arXiv:1704.02571v2 [math.AP].

[4] G. Barles, J. Meireles, On unbounded solutions of ergodic problems in $\mathbb{R}^{m}$ for viscous Hamilton-Jacobi equations, Comm. Partial Differential Equations, 41 (2016), pp. 1985-2003.

[5] G. Barles, A. Porretta, T. Tchamba, On the large time behavior of solutions of the Dirichlet problem for subquadratic viscous Hamilton-Jacobi equations, J. Math. Pures Appl., 94 (2010), pp. 497-519.

[6] G. Barles, P.E. Souganidis, Space-time periodic solutions and long-time behavior of solutions to quasi-linear parabolic equations, SIAM J. Math. Anal., 32 (2001), pp. 1311-1323.

[7] F. Cagnetti, D. Gomes, H. Mitake and H.V. Tran, A new method for large time behavior of degenerate viscous Hamilton-Jacobi equations with convex Hamiltonians,, Ann. Inst. H. Poincaré - AN, 32 (2015), pp. $183-200$

[8] D. Castorina, A. Cesaroni, L. Rossi, Large time behavior of solutions to a degenerate parabolic HamiltonJacobi-Bellman equation, Comm. Pure Applied Analysis, 15 (2016), pp. 1251-1263.

[9] E. Chasseigne, N. Ichihara, Qualitative properties of generalized principal eigenvalues for superquadratic viscous Hamilton-Jacobi equations, Nonlinear Differential Equations Appl. NoDEA, 23:66 (2016), pp. 1-17.

[10] M. Cirant, On the solvability of some ergodic control problems in $\mathbf{R}^{d}$, SIAM J. Control Optim., 52 (2014), pp. 4001-4026.

[11] A. Cosso, M. Fuhrman, H. Pham, Long time asymptotics for fully nonlinear Bellman equations: a backward SDE approach, Stochastic Process. Appl., 126 (2016), pp.1932-1973.

[12] W.H. Fleming, W.M. McEneaney, Risk-sensitive control on an infinite time horizon, SIAM J. Control Optim., 33 (1995), pp. 1881-1915.

[13] W.H. Fleming, M. Soner, Controlled Markov Processes and Viscosity Solutions, Applications of Mathematics 25, Springer-Verlag, New York, 1993.

[14] Y. Fujita, H. Ishii, P. Loreti, Asymptotic solutions of viscous Hamilton-Jacobi equations with OrnsteinUhlenbeck operator, Comm. Partial Differential Equations, 31 (2006), pp. 827-848.

[15] D. Gilbarg, N. S. Trudinger, Elliptic Partial Differential Equations of Second Order, 2nd ed., Grundlehren Math. Wiss. 224, Springer-Verlag, Berlin, 1988.

[16] Y. Hu, P.-Y. Madec, A probabilistic approach to large time behaviour of viscosity solutions of parabolic equations with Neumann boundary conditions, Appl. Math. Optim., 74 (2016), pp. 345-374.

[17] N. Ichihara, Recurrence and transience of optimal feedback processes associated with Bellman equations of ergodic type, SIAM J. Control Optim., 49 (2011), pp. 1938-1960.

[18] N. Ichihara, Large time asymptotic problems for optimal stochastic control with superlinear cost, Stochastic Process. Appl., 122 (2012), pp. 1248-1275.

[19] N. Ichihara, Criticality of viscous Hamilton-Jacobi equations and stochastic ergodic control, J. Math. Pures Appl., 100 (2013), pp. 368-390.

[20] N. Ichihara, The generalized principal eigenvalue for Hamilton-Jacobi-Bellman equations of ergodic type, Ann. I. H. Poincaré - AN, 32 (2015), pp. 623-650.

[21] N. Ichihara, S.J. Sheu, Large time behavior of solutions of Hamilton-Jacobi-Bellman equations with quadratic nonlinearity in gradients, SIAM J. Math. Anal., 45 (2013), pp. 279-306.

[22] H. Kaise, S.J. Sheu, On the structure of solutions of ergodic type Bellman equation related to risk-sensitive control, Ann. Probab., 34 (2005), pp. 284-320.

[23] R.Z. Khasminskii, Stochastic Stability of Differential Equations, 2nd edition, Stochastic Modelling and Applied Probability 66, Springer, 2012.

[24] H. Kunita, Stochastic Flows and Stochastic Differential Equations, Cambridge studies in advanced mathematics $24,1990$.

[25] O. Ley, V. D. Nguyen, Large time behavior for some nonlinear degenerate parabolic equations, J. Math. Pures Appl., 102 (2014), pp. 293-314.

[26] P.-L. Lions, Résolution de problèmes elliptiques quasilinéaires, Arch. Rational Mech. Anal., 74 (1980), pp. 335-353.

[27] O.A. Ladyzhenskaya, N.N. Ural'tseva, Linear and Quasilinear Elliptic Equations, Academic Press, 1968.

[28] H. Nagai, Down side risk minimization via a large deviations approach, Ann. Appl. Probab., 22 (2012), pp. 608-669.

[29] M. Nisio, Stochastic control theory, 2nd ed., Probability Theory and Stochastic Modelling, 72. Springer, Tokyo, 2015.

[30] R.G. Pinsky, Positive Harmonic Functions and Diffusion, Cambridge studies in advanced mathematics 45, 1995.

[31] S. Robertson, H. Xing, Large time behavior of solutions to semilinear equations with quadratic growth in the gradient, SIAM J. Control Optim., 53 (2015), pp. 185-212.

[32] P. Souplet, Q. Zhang, Global solutions of inhomogeneous Hamilton-Jacobi equations, J. Anal. Math., 99 (2006), pp. 355-396. 